\title{
Interaction sums and action of Hecke operators on theta-series
}

\author{
by \\ Anatoli Andrianov (St. Petersburg)
}

To Eberhard Freitag

1. Introduction. One can hardly doubt that the main stream of further progress in number theory goes through the study of representations of Hecke-Shimura algebras of arithmetical discrete subgroups of Lie groups on appropriate spaces of automorphic forms (automorphic representations) and their interaction with each other. An important example of the interaction arises in the case of integral orthogonal and symplectic groups. Recently explicit relations were established in [An06] between zeta functions of integral quadratic forms in two and four variables corresponding to the harmonic eigenvectors of genera 1 and 2, respectively, and symplectic zeta functions of theta-functions weighted by the eigenvectors. The general links of automorphic representations of Hecke-Shimura algebras of orthogonal and symplectic groups as well as underlying relations between the Hecke-Shimura algebras themselves may be provided by transformation formalism, which expresses images of symplectic harmonic theta-functions under the action of Hecke operators through the action of automorph class rings of relevant systems of quadratic forms on harmonic coefficients of the theta-functions. By the transformation formalism we mean an explicit answer to the natural question whether the images of various theta-series and theta-functions of integral quadratic forms under symplectic Hecke operators are again linear combinations of similar theta-series or theta-functions. Unfortunately, the foundation of the formalism (for the last version see [An96]), although quite strong, cannot be considered satisfactory, because it is based in its

2010 Mathematics Subject Classification: Primary 11F27, 11F46, 11F60, 14G10, 20C08. Key words and phrases: interaction sums, harmonic theta-functions, Hecke operators, theta-series.

The author was supported in part by Russian Fund of Fundamental Researches (RFFI), Grant 08-01-00233. 
core on a rather elaborate limit passage exploiting factorization of certain standard polynomials (the Rankin polynomials) over Hecke-Shimura rings of the symplectic group in parabolic extensions (see, e.g., [An79], [An86], [An87]). At the same time, all that is really necessary for an elementary foundation of the formalism is just a simply formulated property of certain finite trigonometric sums, called the interaction sums or iSums. The main purpose of this paper is to analyze relations of iSums with the action of regular Hecke operators on general theta-functions.

The idea to complete a draft of this paper by an elementary section on the action of elements $T^{n}(p)$ appeared during a discussion of the problem with Professor Eberhard Freitag in Heidelberg at the end of 2008. Many years ago Professor Freitag had discovered a quite different and surprisingly simple approach to the explicit transformation formulas for positive definite quadratic forms of level 1 based on the theory of singular modular forms ([Fr81]). It would be desirable to join the advantages of the two approaches and finally close the problem.

Notation. We fix the letters $\mathbb{N}, \mathbb{Z}, \mathbb{Q}$, and $\mathbb{C}$, as usual, for the set of positive rational integers, the ring of rational integers, the field of rational numbers, and the field of complex numbers, respectively.

If $\mathbb{A}$ is a set, $\mathbb{A}_{n}^{m}$ denotes the set of all $m \times n$-matrices with elements in $\mathbb{A}$. If $\mathbb{A}$ is a ring with the identity element, $1_{n}$ denotes the identity element of the ring $\mathbb{A}_{n}^{n}$. The transpose of a matrix $M$ is denoted by ${ }^{t} M$. For two matrices $S$ and $N$ of appropriate sizes we write

$$
S[N]={ }^{t} N S N .
$$

\section{Theta-series and theta-functions of nonsingular quadratic}

forms. The interaction sums are certain trigonometric sums appearing as coefficients in formulas for the action of Hecke operators on theta-functions and theta-series of integral quadratic forms. In this section we shall recall the basic definitions and properties of theta-series and theta-functions.

Let

$$
\mathbf{q}(X)=\frac{1}{2}{ }^{t} X Q X=\frac{1}{2} Q[X] \quad\left({ }^{t} X=\left(x_{1}, \ldots, x_{m}\right)\right)
$$

be a real quadratic form in $m$ variables with matrix $Q={ }^{t} Q$. The form is nonsingular if $\operatorname{det} \mathbf{q}=\operatorname{det} Q \neq 0$. Speaking of quadratic forms, we shall mainly use the equivalent language of their matrices. If the form (2.1) is positive definite, then for $n=1,2, \ldots$ the theta-series $\theta(Z ; Q)$ of $Q$ of genus $n$ is defined to be

$$
\theta(Z ; Q)=\theta^{n}(Z ; Q)=\sum_{N \in \mathbb{Z}_{n}^{m}} \mathbf{e}\{Q[N] Z\}
$$


where the variable $Z$ belongs to the (Siegel) upper half-plane of genus $n$,

$$
\mathbb{H}^{n}=\left\{Z=X+\sqrt{-1} Y \in \mathbb{C}_{n}^{n} \mid{ }^{t} Z=Z, Y>0\right\},
$$

and where we use the abbreviation

$$
\mathbf{e}\{A\}=e^{\pi \sqrt{-1} \sigma(A)}
$$

with $\sigma(A)$ denoting the sum of the diagonal entries of a square matrix $A$. The theta-series is obviously convergent absolutely and uniformly on subsets of $\mathbb{H}^{n}$ of the form

$$
\mathbb{H}_{\varepsilon}^{n}=\left\{Z=X+\sqrt{-1} Y \in \mathbb{H}^{n} \mid Y \geq \varepsilon 1_{n}\right\} \quad \text { with } \varepsilon>0,
$$

and therefore it defines a holomorphic function of $Z$. If, in addition, the form $\mathbf{q}$ is integral, i.e. the matrix $Q$ belongs to the set

$$
\mathbb{E}^{m}=\left\{Q=\left(Q_{i j}\right) \in \mathbb{Z}_{m}^{m} \mid Q_{i j}=Q_{j i}, Q_{i i} \in 2 \mathbb{Z}(i, j=1, \ldots, m)\right\}
$$

of even matrices of order $m$, then the theta-series has Fourier expansion

$$
\theta^{n}(Z ; Q)=\sum_{A \in \mathbb{E}^{n}, A \geq 0} r(A ; Q) \mathbf{e}\{A Z\}
$$

with constant Fourier coefficients expressing the numbers of integral representations of the quadratic form with matrix $A$ by the form $\mathbf{q}$, i.e., the number of solutions of the equation $Q[N]=A$ in integral $m \times n$-matrices $N$.

In the general case, when the form $\mathbf{q}$ may be indefinite, the series (2.2) is not necessarily convergent. Following an approach due to C. L. Siegel (see, e.g., [Si44]), we introduce certain additional variables in the definition of theta-series, which provides their convergence. For a real symmetric nonsingular matrix $Q$ of order $m$, we define the majorant space $\mathbb{M}(Q)$ of $Q$ by

$$
\mathbb{M}(Q)=\left\{H \in \mathbb{R}_{m}^{m} \mid H={ }^{t} H, H>0, H Q^{-1} H=Q\right\} .
$$

The set $\mathbb{M}(Q)$ is a homogeneous space of the real orthogonal group of the form q with matrix $Q$,

$$
O(\mathbf{q})=O(Q)=O_{\mathbb{R}}(Q)=\left\{\left.U \in \mathbb{R}_{m}^{m}\right|^{t} U Q U=Q\right\},
$$

operating on $\mathbb{M}(Q)$ by the rule

$$
O(Q) \ni U: H \mapsto{ }^{t} U H U \quad(H \in \mathbb{M}(Q)) .
$$

Now, for $Z=X+\sqrt{-1} Y \in \mathbb{H}^{n}$ with $n \geq 1$ and $H \in \mathbb{M}(Q)$, we define the theta-series of $Q$ of genus $n$ as a function in variables $Z$ and $H$ given by the series

$$
\Theta(Z ; H, Q)=\Theta^{n}(Z ; H, Q)=\sum_{N \in \mathbb{Z}_{n}^{m}} \mathbf{e}\{Q[N] X+\sqrt{-1} H[N] Y\} .
$$

This series converges absolutely and uniformly on products of the sets (2.4) and compact subsets of $\mathbb{M}(Q)$, and therefore defines a real-analytic function 
on $\mathbb{H}^{n} \times \mathbb{M}(Q)$. If $Q$ is positive definite, then $\mathbb{M}(Q)$ reduces to a single point $H=Q$, and the last theta-series coincides with (2.2).

It turns out to be convenient to add also certain "linear" variables to the definition of theta-series. Let $Q$ be again a real nonsingular symmetric matrix of order $m$, and $H \in \mathbb{M}(Q)$. For $V_{1}, V_{2} \in \mathbb{C}_{n}^{m}$, we define the thetafunction of genus $n$ of the pair $Q, H$ with translations $V=\left(V_{1}, V_{2}\right)$ as a function of the variables $V \in \mathbb{C}_{2 n}^{m}, Z \in \mathbb{H}^{n}$, and $H \in \mathbb{M}(Q)$ given by

$$
\begin{aligned}
& \Theta(V, Z ; H, Q)=\Theta^{n}(V, Z ; H, Q) \\
& =\sum_{N \in \mathbb{Z}_{n}^{m}} \mathbf{e}\left\{Q\left[N-V_{2}\right] X+\sqrt{-1} H\left[N-V_{2}\right] Y+2{ }^{t} V_{1} Q N-{ }^{t} V_{1} Q V_{2}\right\} .
\end{aligned}
$$

The series converges absolutely and uniformly on products of the set (2.4) and compact subsets of $\mathbb{C}_{2 n}^{m} \times \mathbb{M}(Q)$, and so it defines a real-analytic function on $\mathbb{C}_{2 n}^{m} \times \mathbb{H}^{n} \times \mathbb{M}(Q)$.

Finally, in certain arithmetical applications there appear theta-functions of a more general kind, namely theta-functions with harmonic coefficients defined by the series

$$
\Theta_{P}(V, Z ; H, Q)=\Theta_{P}^{n}(V, Z ; H, Q)
$$

$=\sum_{N \in \mathbb{Z}_{n}^{m}} P\left(N-V_{2}\right) \mathbf{e}\left\{Q\left[N-V_{2}\right] X+\sqrt{-1} H\left[N-V_{2}\right] Y+2{ }^{t} V_{1} Q N-{ }^{t} V_{1} Q V_{2}\right\}$,

where $P$ is a vector-valued polynomial harmonic form relative to the pair $(Q, H)$ in the sense of $[\mathrm{An} 95, \S 4]$ or $[\mathrm{An} 96, \S 1]$. If the $P$ factor is identically equal to 1 , the theta-function (2.8) turns into (2.7).

To simplify notation we shall restrict our considerations to the onedimensional case of theta-functions with polynomial-valued harmonic factors, although the main results remains true for vector-valued harmonic factors (see [An96]). First we briefly recall the relevant definitions. A polynomial function $P: \mathbb{C}_{n}^{m} \rightarrow \mathbb{C}$ is said to be a harmonic form of weight $d$ if it is harmonic in $m n$ variables in the sense that

$$
\sum_{i, j} \frac{\partial^{2} P(X)}{\left(\partial x_{i j}\right)^{2}}=0
$$

and satisfies the condition

$$
P(X A)=(\operatorname{det} A)^{d} P(X) \quad \text { for all } A \in \mathrm{GL}_{n}(\mathbb{C}) .
$$

Let $\mathbf{q}$ be a nonsingular quadratic form (2.1) with matrix $Q$, and let $H \in$ $\mathbb{M}(Q)$. Since $H$ is positive definite and $Q^{-1}[H]=Q$, there exists a real invertible matrix $S$ of order $m$ such that

$$
H={ }^{t} S S \text { and } Q={ }^{t} S\left(\begin{array}{cc}
1_{s} & 0 \\
0 & -1_{l}
\end{array}\right) S
$$


where $(s, l)$ is the signature of $\mathbf{q}$. We set $S=\left(\begin{array}{c}S_{+} \\ S_{-}\end{array}\right)$with $S_{+} \in \mathbb{R}_{m}^{s}$ and $S_{-} \in \mathbb{R}_{m}^{l}$. If $P_{+}$is a harmonic form of degree $d$ on $\mathbb{C}_{s}^{m}$ and $P_{-}$is a harmonic form of degree $d^{\prime}$ on $\mathbb{C}_{l}^{m}$, we say that the polynomial function $P=P_{Q, H}$ on $\mathbb{C}_{n}^{m}$ defined by

$$
\mathbb{C}_{n}^{m} \ni X \mapsto P(X)=P_{+}\left(S_{+} X\right) P_{-}\left(S_{-} X\right)
$$

is a harmonic form of weight $\left(d, d^{\prime}\right)$ relative to the pair $Q, H$. It follows from the definition that the harmonic form $P$ of weight $\left(d, d^{\prime}\right)$ satisfies

$$
P(X A)=(\operatorname{det} A)^{d+d^{\prime}} P(X) \quad \text { for all } A \in \mathrm{GL}_{n}(\mathbb{C}) .
$$

According to [An95, Theorems 4.1-4.3], we can formulate the following theorem.

THEOREM 2.1. Let $Q$ be an even nonsingular matrix of even order $m=$ $2 k$ with signature $(s, l)$, let $q$ be the level of $Q$, i.e., the least $q \in \mathbb{N}$ such that the matrix $q Q^{-1}$ is even, and let $H \in \mathbb{M}(Q)$. Then the theta-function (2.7) of $Q$ of genus $n \geq 1$ satisfies the functional equation

$$
\Theta\left(V \cdot{ }^{t} M, M\langle Z\rangle ; H, Q\right)=j_{Q}(M, Z) \Theta(V, Z ; H, Q)
$$

for each matrix $M=\left(\begin{array}{ll}A & B \\ C & D\end{array}\right)$ in the group

$$
\Gamma_{0}^{n}(q)=\left\{M=\left(\begin{array}{ll}
A & B \\
C & D
\end{array}\right) \in \operatorname{Sp}_{n}(\mathbb{Z}) \mid C \equiv 0(\bmod q)\right\},
$$

where $M\langle Z\rangle=\left(\begin{array}{cc}A & B \\ C & D\end{array}\right)\langle Z\rangle=(A Z+B)(C Z+D)^{-1}$,

$$
j_{Q}(M, Z)=\chi_{Q}(\operatorname{det} D)(\operatorname{det}(C Z+D))^{(s-l) / 2}|\operatorname{det}(C Z+D)|^{l},
$$

and $\chi_{Q}$ is the character of the quadratic form with matrix $Q$.

More generally, under the same assumptions, let $P$ be a polynomial harmonic form of weight $\left(d, d^{\prime}\right)$ relative to the pair $Q, H$. Then the thetafunction (2.8) satisfies the functional equation

$$
\Theta_{P}\left(V \cdot{ }^{t} M, M\langle Z\rangle ; H, Q\right)=j_{Q, P}(M, Z) \Theta_{P}(V, Z ; H, Q)
$$

for each $M=\left(\begin{array}{cc}A & B \\ C & D\end{array}\right) \in \Gamma_{0}^{n}(q)$, where

$$
j_{Q, P}(M, Z)=j_{Q}(M, Z) \operatorname{det}(C Z+D)^{d} \operatorname{det}(C \bar{Z}+D)^{d^{\prime}}
$$

and $\bar{Z}$ is the complex conjugate matrix of $Z$.

We recall here that the character of an integral nonsingular quadratic form in an even number $m$ of variables with matrix $Q$ of level $q$ is the Dirichlet character modulo $q$ satisfying the conditions

$$
\begin{gathered}
\chi_{Q}(-1)=(-1)^{m / 2}, \\
\chi_{Q}(p)=\left(\frac{(-1)^{m / 2} \operatorname{det} Q}{p}\right) \quad(\text { the Legendre symbol) }
\end{gathered}
$$


if $p$ is an odd prime not dividing $q$, and

$$
\chi_{Q}(2)=2^{-m / 2} \sum_{R \in \mathbb{Z}^{m} / 2 \mathbb{Z}^{m}} \mathbf{e}\left\{\frac{1}{2} Q[R]\right\}
$$

if $q$ is odd.

3. Symplectic Hecke operators. Following the general pattern of the theory of Hecke operators on modular forms (see, e.g., [An87, Ch. 4], or $[$ An96, §2]), we now recall the basic definitions and simplest properties of (regular) Hecke-Shimura rings and Hecke operators for the groups $\Gamma_{0}^{n}(q)$. Let us denote by

$$
\mathcal{H}_{0}^{n}(q)=\mathcal{H}\left(\Gamma_{0}^{n}(q), \Sigma_{0}^{n}(q)\right)
$$

the Hecke-Shimura ring of the semigroup

$$
\begin{aligned}
\Sigma_{0}^{n}(q)=\{M= & \left(\begin{array}{ll}
A & B \\
C & D
\end{array}\right) \in \mathbb{Z}_{2 n}^{2 n} \mid{ }^{t} M J_{n} M=\mu(M) J_{n}, \mu(M)>0, \\
& \quad \operatorname{gcd}(\operatorname{det} M, q)=1, C \equiv 0(\bmod q)\} \quad\left(J_{n}=\left(\begin{array}{cc}
0 & 1_{n} \\
-1_{n} & 0
\end{array}\right)\right)
\end{aligned}
$$

relative to the group $\Gamma_{0}^{n}(q)$ (over $\mathbb{C}$ ). The ring $\mathcal{H}_{0}^{n}(q)$ consists of all formal finite linear combinations with complex coefficients of the symbols $\tau(M)$, which are in one-to-one correspondence with double cosets $\Gamma_{0}^{n}(q) M \Gamma_{0}^{n}(q) \subset$ $\Sigma_{0}^{n}(q)$. It is convenient to write each of the symbols $\tau(M)$, also called the double cosets, as the formal sum of the different left cosets it contains (more precisely, of the corresponding symbols),

$$
\tau(M)=\sum_{M^{\prime} \in \Gamma \backslash \Gamma M \Gamma}\left(\Gamma M^{\prime}\right) \quad\left(\Gamma=\Gamma_{0}^{n}(q), M \in \Sigma_{0}^{n}(q)\right) .
$$

Then each element $T \in \mathcal{H}_{0}^{n}(q)$ can also be written as the formal linear combination of different left cosets,

$$
T=\sum_{\alpha} c_{\alpha}\left(\Gamma_{0}^{n}(q) M_{\alpha}\right) \quad\left(c_{\alpha} \in \mathbb{C}\right) .
$$

These linear combinations can be characterized by the condition of invariance with respect to right multiplication by elements of $\Gamma_{0}^{n}(q)$ :

$$
T \gamma=\sum_{\alpha} c_{\alpha}\left(\Gamma_{0}^{n}(q) M_{\alpha} \gamma\right)=T \quad \text { for all } \gamma \in \Gamma_{0}^{n}(q) .
$$

In this notation, the product in $\mathcal{H}_{0}^{n}(q)$ can be defined by

$$
T T^{\prime}=\sum_{\alpha} c_{\alpha}\left(\Gamma_{0}^{n}(q) M_{\alpha}\right) \sum_{\beta} c_{\beta}^{\prime}\left(\Gamma_{0}^{n}(q) M_{\beta}^{\prime}\right)=\sum_{\alpha, \beta} c_{\alpha} c_{\beta}^{\prime}\left(\Gamma_{0}^{n}(q) M_{\alpha} M_{\beta}^{\prime}\right) .
$$


The ring $\mathcal{H}_{0}^{n}(q)$ is a commutative $\mathbb{C}$-algebra generated over $\mathbb{C}$ by a countable set of algebraically independent elements. As a set of algebraically independent generators one can take, for example, double cosets of the form

$$
\left\{\begin{array}{l}
T^{n}(p)=\tau(\operatorname{diag}(\underbrace{1, \ldots, 1}_{n}, \underbrace{p, \ldots, p}_{n})), \\
T_{i}^{n}\left(p^{2}\right)=\tau\left(\operatorname{diag}(\underbrace{1, \ldots, 1}_{n-i}, \underbrace{p, \ldots, p}_{i}, \underbrace{p^{2}, \ldots, p^{2}}_{n-i}, \underbrace{p, \ldots, p}_{i})\right)
\end{array}\right.
$$

$(1 \leq i \leq n)$,

where $p$ runs over all prime numbers not dividing $q$ (see [An87, Theorem 3.3.23]).

In order to define Hecke operators on theta-functions and theta-series we introduce certain linear spaces containing theta-functions. For fixed $m, n \in \mathbb{N}$ we denote by $\mathfrak{F}=\mathfrak{F}_{m, n}$ the space of all complex-valued real-analytic functions

$$
F=F(V, Z): \mathbb{C}_{2 n}^{m} \times \mathbb{H}^{n} \rightarrow \mathbb{C}
$$

and define an action of the semigroup $\Sigma_{0}^{n}(q)$ on these spaces by the Petersson operators

$$
\Sigma_{0}^{n}(q) \ni M: F=\left.F(V, Z) \mapsto F\right|_{\mathbf{j}} M=j_{Q, P}(M, Z)^{-1} F\left(V^{t} M, M\langle Z\rangle\right),
$$

where $\mathbf{j}=j_{Q, P}(M, Z)$ is defined by (2.14). It is well-known that the function $\operatorname{det}(C Z+D)$ does not vanish on $\Sigma_{0}^{n}(q) \times \mathbb{H}^{n}$ and hence neither does any of the functions $j_{Q, P}(M, Z)$. If $M=\left(\begin{array}{ll}A & B \\ C & D\end{array}\right)$ and $M_{1}=\left(\begin{array}{ll}A_{1} & B_{1} \\ C_{1} & D_{1}\end{array}\right)$ belong to $\Sigma_{0}^{n}(q)$ and $M^{\prime}=\left(\begin{array}{cc}A^{\prime} & B^{\prime} \\ C^{\prime} & D^{\prime}\end{array}\right)=M M_{1}$, then an easy direct computation shows that

$$
\left(C \cdot M_{1}\langle Z\rangle+D\right)\left(C_{1} Z+D_{1}\right)=C^{\prime} Z+D^{\prime} \quad\left(Z \in \mathbb{H}^{n}\right),
$$

and

$$
\begin{aligned}
\chi_{Q}\left(\operatorname{det} D^{\prime}\right) & =\chi_{Q}\left(\operatorname{det}\left(C B_{1}+D D_{1}\right)\right)=\chi_{Q}\left(\operatorname{det} D D_{1}\right) \\
& =\chi_{Q}(\operatorname{det} D) \chi_{Q}\left(\operatorname{det} D_{1}\right),
\end{aligned}
$$

since $C \equiv 0_{n}(\bmod q)$. These relations imply that the functions $j_{Q, P}(M, Z)$ satisfy the relations of automorphic factors, i.e.,

$$
j_{Q, P}\left(M, M_{1}\langle Z\rangle\right) j_{Q, P}\left(M_{1}, Z\right)=j_{Q, P}\left(M M_{1}, Z\right)
$$

for all $M, M_{1} \in \Sigma_{0}^{n}(q), Z \in \mathbb{H}^{n}$. This implies that the Petersson operators map the space $\mathfrak{F}_{n, m}$ into itself and satisfy the rule

$$
\left.\left.F\right|_{\mathbf{j}} M\right|_{\mathbf{j}} M_{1}=\left.F\right|_{\mathbf{j}} M M_{1} \quad\left(F \in \mathfrak{F}_{n, m}, M, M_{1} \in \Sigma_{0}^{n}(q)\right) .
$$

It allows us to define the standard representation $\left.T \mapsto\right|_{\mathbf{j}} T$ of the HeckeShimura ring $\mathcal{H}_{0}^{n}(q)=\mathcal{H}\left(\Gamma_{0}^{n}(q), \Sigma_{0}^{n}(q)\right)$ on the subspace

$$
\begin{aligned}
\mathfrak{F}\left(\Gamma_{0}^{n}(q)\right) & =\mathfrak{F}_{n, m}\left(\Gamma_{0}^{n}(q)\right) \\
& =\left\{F \in \mathfrak{F}_{n, m}|F|_{\mathbf{j}} \gamma=F \text { for all } \gamma \in \Gamma_{0}^{n}(q)\right\}
\end{aligned}
$$


of all $\Gamma_{0}^{n}(q)$-invariant functions by Hecke operators: the Hecke operator $\left.\right|_{\mathbf{j}} T$ on the space $\mathfrak{F}\left(\Gamma_{0}^{n}(q)\right)$ corresponding to an element of the form (3.2) is defined by

$$
\left.F\right|_{\mathbf{j}} T=\left.\sum_{\alpha} c_{\alpha} F\right|_{\mathbf{j}} M_{\alpha} \quad\left(F=F(V, Z) \in \mathfrak{F}_{n, m}\left(\Gamma_{0}^{n}(q)\right)\right),
$$

where $\left.\right|_{\mathbf{j}} M_{\alpha}$ are the Petersson operators (3.4) corresponding to $\mathbf{j}=j_{Q, P}^{n}(M, Z)$. The Hecke operators are independent of the choice of the representatives $M_{\alpha} \in \Gamma_{0}^{n}(q) M_{\alpha}$, and map $\mathfrak{F}_{n, m}\left(\Gamma_{0}^{n}(q)\right)$ into itself. It follows from the definition of multiplication in the Hecke-Shimura rings and (3.5) that the Hecke operators satisfy

$$
\left.{ }_{\mathbf{j}} T\right|_{\mathbf{j}} T^{\prime}={ }_{\mathbf{j}} T T^{\prime} \quad \text { for all } T, T^{\prime} \in \mathcal{H}_{0}^{n}(q) .
$$

Hence, the map $\left.T \mapsto\right|_{\mathbf{j}} T$ is a linear representation of the ring $\mathcal{H}_{0}^{n}(q)$ on the space $\mathfrak{F}_{n, m}\left(\Gamma_{0}^{n}(q)\right)$.

In the particular case of the trivial harmonic factor $P=1$ of weight $\left(d_{+}, d_{-}\right)=(0,0)$, we obtain the Petersson operators and Hecke operators with the automorphic factor $\mathbf{j}=j_{Q}(M, Z)$ of the form (2.12).

In the notation and under the assumptions of Theorem 2.1, the thetafunctions $\Theta_{P}^{n}(V, Z ; H, Q)$ and $\Theta^{n}(V, Z ; H, Q)=\Theta_{1}^{n}(V, Z ; H, Q)$, viewed as functions of $V$ and $Z$, belong to the space $\mathfrak{F}_{n, m}\left(\Gamma_{0}^{n}(q)\right)$. The target of this paper is to establish when the images of the theta-functions under the Hecke operators can be written as finite linear combinations with constant coefficients of similar theta-functions. We shall see later that the general case of an arbitrary harmonic form $P$ can usually be reduced to the particular case of the trivial form $P=1$. First we shall express these images as infinite sums with explicitly written coefficients.

We begin with two technical remarks. By definition, each matrix $M \in$ $\Sigma_{0}^{n}(q)$ satisfies the relation ${ }^{t} M J_{n} M=\mu(M) J_{n}$, where $\mu(M)$ is a positive integer coprime with $q$. The number $\mu(M)$ is called the multiplier of $M$. Clearly,

$$
\mu\left(M M^{\prime}\right)=\mu(M) \mu\left(M^{\prime}\right) \quad\left(M, M^{\prime} \in \Sigma_{0}^{n}(q)\right), \quad \mu(M)=1 \Leftrightarrow M \in \Gamma_{0}^{n}(q) .
$$

It follows that the function $M \mapsto \mu(M)$ takes a constant value on each left and double coset of $M$ modulo the group $\Gamma_{0}^{n}(q)$. Hence, one can speak of the multiplier of the corresponding cosets, $\mu\left(\Gamma_{0}^{n}(q) M\right)=\mu\left(\Gamma_{0}^{n}(q) M \Gamma_{0}^{n}(q)\right)=$ $\mu(M)$. We shall say that a nonzero formal finite linear combination $T$ of left or double cosets modulo $\Gamma_{0}^{n}(q)$ of matrices in $\Sigma_{0}^{n}(q)$ is homogeneous with multiplier $\mu(T)=\mu$ if all cosets entering $T$ with nonzero coefficients have the same multiplier $\mu$. It is clear that each finite linear combination of cosets is a sum of homogeneous combinations with different multipliers, called homogeneous components, and these components are uniquely deter- 
mined. This allows us, in particular, to reduce the study of arbitrary Hecke operators $\mid T$ to the case of $T$ homogeneous.

Another reduction is related to a specific choice of representatives in the left cosets $\Gamma_{0}^{n}(q) M \subset \Sigma_{0}^{n}(q)$. According, for example, to [An87, Lemma 3.3.4], each of the left cosets contains a representative of the form

$$
M=\left(\begin{array}{cc}
A & B \\
0 & D
\end{array}\right) \quad \text { with } A, B, D \in \mathbb{Z}_{n}^{n},{ }^{t} A D=\mu(M) 1_{n},{ }^{t} B D={ }^{t} D B .
$$

Such representatives are convenient for computing the action of Hecke operators and will be referred to as triangular representatives.

Proposition 3.1. Let $Q$ be an even nonsingular matrix of even order $m=2 k$ with signature $(s, l)$ and level $q, H \in \mathbb{M}(Q)$, and let $P$ be a polynomial harmonic form of weight $\left(d, d^{\prime}\right)$ relative to the pair $Q, H$. Then the image

$$
\begin{aligned}
\left.\Theta_{P}^{n}(V, Z ; H, Q)\right|_{\mathbf{j}} T & =\left.\sum_{\alpha} c_{\alpha} \Theta_{P}^{n}(V, Z ; H, Q)\right|_{\mathbf{j}} M_{\alpha} \\
= & \sum_{\alpha} c_{\alpha} j_{Q, P}\left(M_{\alpha}, Z\right)^{-1} \Theta_{P}\left(V \cdot{ }^{t} M_{\alpha}, M_{\alpha}\langle Z\rangle ; H, Q\right)
\end{aligned}
$$

of the theta-function $\Theta_{P}^{n}(V, Z ; H, Q) \in \mathfrak{F}_{n, m}\left(\Gamma_{0}^{n}(q)\right)$, considered as a function of $V=\left(V_{1}, V_{2}\right)$ and $Z$, under the action of the Hecke operator (3.7) corresponding to a homogeneous element $T \in \mathcal{H}_{0}^{n}(q)$ of the form (3.2) with multiplier $\mu(T)=\mu\left(M_{\alpha}\right)=\mu$ and with triangular representatives $M_{\alpha}$,

$$
M_{\alpha}=\left(\begin{array}{cc}
A_{\alpha} & B_{\alpha} \\
0 & D_{\alpha}
\end{array}\right) \quad\left(A_{\alpha}, B_{\alpha}, D_{\alpha} \in \mathbb{Z}_{n}^{n},{ }^{t} A_{\alpha} D_{\alpha}=\mu 1_{n},{ }^{t} B_{\alpha} D_{\alpha}={ }^{t} D_{\alpha} B_{\alpha}\right),
$$

can be written in the form

$$
\begin{aligned}
\Theta_{P}^{n}\left(\left(V_{1}, V_{2}\right), X\right. & +\sqrt{-1} Y ; H, Q)\left.\right|_{\mathbf{j}} T \\
= & \sum_{N \in C^{n}(Q / \mu)} I(N, Q, T) P\left(\mu^{-1}\left(N-\mu V_{2}\right)\right) \\
& \times \mathbf{e}\left\{\mu^{-1} Q\left[N-\mu V_{2}\right] X+\sqrt{-1} \mu^{-1} H\left[N-\mu V_{2}\right] Y\right. \\
& \left.+2^{t}\left(\mu V_{1}\right) \mu^{-1} Q N-{ }^{t}\left(\mu V_{1}\right) \mu^{-1} Q\left(\mu V_{2}\right)\right\},
\end{aligned}
$$

where

$$
\begin{aligned}
C^{n}(Q / \mu) & =\left\{N \in \mathbb{Z}_{n}^{m} \mid \mu^{-1} Q[N] \in \mathbb{E}^{n}\right\} \\
I(N, Q, T) & =\sum_{\alpha, N \cdot{ }^{t} D_{\alpha} \equiv 0(\bmod \mu)} c_{\alpha} j_{Q}\left(D_{\alpha}\right)^{-1} \mathbf{e}\left\{\mu^{-2} Q[N] \cdot{ }^{t} D_{\alpha} B_{\alpha}\right\}, \\
j_{Q}\left(D_{\alpha}\right) & =\chi_{Q}\left(\left|\operatorname{det} D_{\alpha}\right|\right)\left|\operatorname{det} D_{\alpha}\right|^{m / 2} .
\end{aligned}
$$


Proof. We follow the proof of [An96, Proposition 2.1] with a minor correction at the end. For brevity, we introduce the notation

$$
\begin{aligned}
& \mathbf{e}\{V, Z, H, Q ; N\} \\
& \quad=\mathbf{e}\left\{Q\left[N-V_{2}\right] X+\sqrt{-1} H\left[N-V_{2}\right] Y+2{ }^{t} V_{1} Q N-{ }^{t} V_{1} Q V_{2}\right\}
\end{aligned}
$$

for the exponential factor in the generic term of the series (2.8), where $V=\left(V_{1}, V_{2}\right)$. An easy direct calculation shows that these exponents satisfy the identity

$$
\begin{aligned}
\mathbf{e}\left\{V^{t} M, M\langle Z\rangle, H, Q ; N\right\} & \\
& =\mathbf{e}\left\{B D^{-1} Q[N]\right\} \mathbf{e}\left\{\mu V, Z, \mu^{-1} H, \mu^{-1} Q ; N A\right\}
\end{aligned}
$$

for each matrix $M$ of the triangular form (3.9).

By (3.4) in notation (3.15), we obtain

$$
\begin{gathered}
\left.\Theta_{P}(V, Z ; H, Q)\right|_{\mathbf{j}} T=\left.\sum_{\alpha} c_{\alpha} \Theta_{P}(V, Z ; H, Q)\right|_{\mathbf{j}}\left(\begin{array}{cc}
A_{\alpha} & B_{\alpha} \\
0 & D_{\alpha}
\end{array}\right) \\
=\sum_{N \in \mathbb{Z}_{n}^{m}} \sum_{\alpha} c_{\alpha} j_{Q, P}\left(M_{\alpha}, Z\right)^{-1} P\left(N-V_{2} \cdot{ }^{t} D_{\alpha}\right) \mathbf{e}\left\{V^{t} M_{\alpha}, M_{\alpha}\langle Z\rangle, H, Q ; N\right\} .
\end{gathered}
$$

Since, by (2.14), (2.12), and (2.15), we can write

$$
\begin{aligned}
j_{Q, P}\left(M_{\alpha}, Z\right) & =\chi_{Q}\left(\operatorname{det} D_{\alpha}\right)\left(\operatorname{det} D_{\alpha}\right)^{(s-l) / 2}\left|\operatorname{det} D_{\alpha}\right|^{l} \operatorname{det}\left(D_{\alpha}\right)^{d} \operatorname{det}\left(D_{\alpha}\right)^{d^{\prime}} \\
& =\chi\left(\left|\operatorname{det} D_{\alpha}\right|\right)\left|\operatorname{det} D_{\alpha}\right|^{m / 2} \operatorname{det}\left(D_{\alpha}\right)^{d+d^{\prime}}=j_{Q}\left(D_{\alpha}\right) \operatorname{det}\left(D_{\alpha}\right)^{d+d^{\prime}},
\end{aligned}
$$

it follows, by $(2.9)$, that

$$
\begin{array}{rl}
j_{Q, P}\left(M_{\alpha}, Z\right)^{-1} & P\left(N-V_{2}{ }^{t} D_{\alpha}\right) \\
& =j_{Q}\left(D_{\alpha}\right)^{-1} \operatorname{det}\left(D_{\alpha}\right)^{-\left(d+d^{\prime}\right)} P\left(\mu^{-1} N A_{\alpha}{ }^{t} D_{\alpha}-V_{2}{ }^{t} D_{\alpha}\right) \\
& =j_{Q}\left(D_{\alpha}\right)^{-1} P\left(\mu^{-1} N A_{\alpha}-V_{2}\right) .
\end{array}
$$

Hence, by using (3.16) with $M=M_{\alpha}$, we can rewrite the sum in (3.17) as

$$
\begin{aligned}
& \sum_{N \in \mathbb{Z}_{n}^{m}} \sum_{\alpha} c_{\alpha} j_{Q}\left(D_{\alpha}\right)^{-1} \mathbf{e}\left\{B_{\alpha} D_{\alpha}^{-1} Q[N]\right\} P\left(\mu^{-1}\left(N A_{\alpha}-\mu V_{2}\right)\right) \\
& \times \mathbf{e}\left\{\mu V, Z, \mu^{-1} H, \mu^{-1} Q ; N A_{\alpha}\right\} .
\end{aligned}
$$

Collecting all the terms with a fixed matrix $N A_{\alpha}=\mu N^{t} D_{\alpha}^{-1}=\widetilde{N} \in \mathbb{Z}_{n}^{m}$ and using the obvious relation

$$
\mathbf{e}\left\{B_{\alpha} D_{\alpha}^{-1} Q[N]\right\}=\mathbf{e}\left\{D_{\alpha}^{-1} Q\left[\mu^{-1} \widetilde{N}^{t} D_{\alpha}\right] B_{\alpha}\right\}=\mathbf{e}\left\{\mu^{-2} Q[\widetilde{N}]^{t} D_{\alpha} B_{\alpha}\right\},
$$

we get the relation

$$
\begin{aligned}
\left.\Theta_{P}(V, Z ; H, Q)\right|_{\mathbf{j}} T= & \sum_{\widetilde{N} \in \mathbb{Z}_{n}^{m}}\left\{\sum_{\alpha, \widetilde{N} A_{\alpha}^{-1} \in \mathbb{Z}_{n}^{m}} c_{\alpha} j_{Q}\left(D_{\alpha}\right)^{-1} \mathbf{e}\left\{\mu^{-2} Q[\widetilde{N}]^{t} D_{\alpha} B_{\alpha}\right\}\right\} \\
& \times P\left(\mu^{-1}\left(\widetilde{N}-\mu V_{2}\right)\right) \mathbf{e}\left\{\mu V, Z, \mu^{-1} H, \mu^{-1} Q ; \widetilde{N}\right\} .
\end{aligned}
$$


Hence, since the condition $\widetilde{N} A_{\alpha}^{-1} \in \mathbb{Z}_{n}^{m}$ is clearly equivalent to the condition $\widetilde{N}^{t} D_{\alpha} \equiv 0(\bmod \mu)$, the sum in parentheses coincides with $I(\widetilde{N}, Q, T)$, and by omitting tildes, we obtain the formula

$$
\begin{aligned}
\left.\Theta_{P}(V, Z ; H, Q)\right|_{\mathbf{j}} T & \\
= & \sum_{N \in \mathbb{Z}_{n}^{m}} I(N, Q, T) P\left(\mu^{-1}\left(N-\mu V_{2}\right)\right) \mathbf{e}\left\{\mu V, Z, \mu^{-1} H, \mu^{-1} Q ; N\right\} \\
= & \sum_{N \in \mathbb{Z}_{n}^{m}} I(N, Q, T) P\left(\mu^{-1}\left(N-\mu V_{2}\right)\right) \mathbf{e}\left\{\mu^{-1} Q\left[N-\mu V_{2}\right] X\right. \\
& \left.\quad+\sqrt{-1} \mu^{-1} H\left[N-\mu V_{2}\right] Y+2^{t}\left(\mu V_{1}\right) \mu^{-1} Q N-{ }^{t}\left(\mu V_{1}\right) \mu^{-1} Q\left(\mu V_{2}\right)\right\} .
\end{aligned}
$$

To complete the proof it remains to show that

$$
I(N, Q, T)=0 \quad \text { if } N \in \mathbb{Z}_{n}^{m} \text {, but } N \notin C^{n}(Q / \mu) .
$$

To this end, we use the last relation for the case of $P$ identically equal to 1, i.e. for the theta-function (2.7). In this case, since Hecke operators map the corresponding subspace of $\Gamma_{0}^{n}(q)$-invariant functions into itself, we conclude that the right-hand side of the relation is invariant with respect to every Petersson operator $\left.\right|_{\mathbf{j}}\left(\begin{array}{cc}1_{n} & B \\ 0 & 1_{n}\end{array}\right)$ with $\mathbf{j}=j_{Q}(M, Z)$ of the form (2.12) and $B={ }^{t} B \in \mathbb{Z}_{n}^{n}$, which transforms a function $F\left(\left(V_{1}, V_{2}\right), Z\right)$ to the function $F\left(\left(V_{1}+V_{2} B, V_{2}\right), Z+B\right)$. In particular, by taking $V_{2}=0$, we get

$$
\begin{array}{rl}
\sum_{N \in \mathbb{Z}_{n}^{m}} & I(N, Q, T) \mathbf{e}\left\{\mu^{-1} Q[N] X+\sqrt{-1} \mu^{-1} H[N] Y+2{ }^{t}\left(\mu V_{1}\right) \mu^{-1} Q N\right\} \\
= & \sum_{N \in \mathbb{Z}_{n}^{m}} I(N, Q, T) \mathbf{e}\left\{\mu^{-1} Q[N](X+B)+\sqrt{-1} \mu^{-1} H[N] Y+2{ }^{t} V_{1} Q N\right\} \\
= & \sum_{N \in \mathbb{Z}_{n}^{m}} I(N, Q, T) \mathbf{e}\left\{\mu^{-1} Q[N] B\right\} \\
& \times \mathbf{e}\left\{\mu^{-1} Q[N] X+\sqrt{-1} \mu^{-1} H[N] Y+2{ }^{t} V_{1} Q N\right\} .
\end{array}
$$

Hence, by the uniqueness of the Fourier expansion of the analytical and periodic (in $V_{1}$ ) function $\left.\Theta\left(\left(V_{1}, 0\right), Z ; H, Q\right)\right|_{\mathbf{j}} T$, we obtain the equality of the corresponding Fourier coefficients,

$$
\begin{aligned}
& I(N, Q, T) \mathbf{e}\left\{\mu^{-1} Q[N] X+\sqrt{-1} \mu^{-1} H[N] Y\right\} \\
& \quad=I(N, Q, T) \mathbf{e}\left\{\mu^{-1} Q[N] B\right\} \mathbf{e}\left\{\mu^{-1} Q[N] X+\sqrt{-1} \mu^{-1} H[N] Y\right\}
\end{aligned}
$$

for all $N \in \mathbb{Z}_{n}^{n}$. If $\mu^{-1} Q[N] \notin \mathbb{E}^{n}$, then, clearly, there is an integral symmetric matrix $B$ of order $n$ such that $\sigma\left(\mu^{-1} Q[N] B\right) \notin 2 \mathbb{Z}$ and so $\left\{\mu^{-1} Q[N] B\right\}$ $\neq 1$. The relations (3.18) and formula (3.11) follow.

We call the trigonometric sums $I(N, Q, T)$ defined for homogeneous $T \in \mathcal{H}_{0}^{n}(q)$ by $(2.13)$ and extended by linearity on the whole of $\mathcal{H}_{0}^{n}(q)$ the 
interaction sums (or, in a modern fashion, just iSums) (of genus n), because the sums link certain structures related to orthogonal and symplectic groups (see, e.g., [An96]).

Of the elementary properties of iSums we record here only the following simple lemma.

Lemma 3.2. The iSums $I(N, Q, T)$ for $N \in C^{n}(Q / \mu)$ and $T \in \mathcal{H}_{0}^{n}(q)$ are independent of the choice of triangular representatives in expansions (3.2) of $T$ and satisfy the relations

$$
I(\lambda N \gamma, Q, T)=I(N, Q[\lambda], T) \quad \text { for all } \lambda \in \mathrm{GL}_{m}(\mathbb{Z}) \text { and } \gamma \in \mathrm{GL}_{n}(\mathbb{Z}) .
$$

Proof. The independence of the choice of representatives easily follows from the definitions. If $T$ is a homogeneous element of the form (3.2) with triangular representatives $M_{\alpha}$ satisfying $\mu\left(M_{\alpha}\right)=\mu(T)=\mu$, then by (3.13) we obtain

$$
\begin{aligned}
& I(\lambda N \gamma, Q, T) \\
& =\sum_{\alpha, \lambda N^{t}\left(D_{\alpha}{ }^{t} \gamma\right) \equiv 0(\bmod \mu)} c_{\alpha} j_{Q}\left(D_{\alpha}{ }^{t} \gamma\right)^{-1} \mathbf{e}\left\{\mu^{-2} Q[\lambda][N] \cdot{ }^{t}\left(D_{\alpha}{ }^{t} \gamma\right) B_{\alpha}{ }^{t} \gamma\right\} \\
& \quad=I\left(N, Q[\lambda], T\left(\begin{array}{cc}
\gamma^{-1} & 0 \\
0 & t_{\gamma}
\end{array}\right)\right)=I(N, Q[\lambda], T),
\end{aligned}
$$

since $T$ is invariant under right multiplication by elements of $\Gamma_{0}^{n}(q)$.

\section{Siegel operator and Zharkovskaya mapping on theta-func-} tions. The Zharkovskaya mapping and commutation relations for Hecke operators and Siegel operators were originally introduced in the situation of holomorphic modular forms of integral weight for congruence subgroups of the symplectic modular group $\Gamma^{n}=\operatorname{Sp}_{n}(\mathbb{Z})$ (see, e.g., [An87, §4.2.4]). Here we shall use as a model the standard definitions of the Siegel operator $\Phi$ and the Zharkovskaya map $\Psi$ acting on theta-functions of integral quadratic forms. This will allow us to link the action of Hecke operators on theta-functions of different genera and the corresponding interaction sums. It should be noted that although the proofs for theta-functions are similar to those for modular forms, the final formulas can differ due to different customary normalization of Hecke operators.

Throughout this section, $Q$ is the matrix of an integral nonsingular quadratic form $\mathbf{q}(X)$ in an even number of variables $m=2 k,(s, l)$ is the signature of $\mathbf{q}, q$ the level of $Q$, and $\chi_{Q}$ the corresponding Dirichlet character modulo $q$.

For a function $F: \mathbb{C}_{2 n}^{m} \times \mathbb{H}^{n} \rightarrow \mathbb{C}$ and $0 \leq r \leq n$, we define the Siegel operator $\Phi^{n, r}$ by 


$$
\begin{aligned}
\left(F \mid \Phi^{n, r}\right) & \left(V^{\prime}, Z^{\prime}\right) \\
= & \lim _{\lambda \rightarrow+\infty} F\left(\left(V_{1}^{\prime}, 0_{n-r}^{m}, V_{2}^{\prime}, 0_{n-r}^{m}\right),\left(\begin{array}{cc}
Z^{\prime} & 0_{n-r}^{r} \\
0_{r}^{n-r} & \sqrt{-1} \lambda \cdot 1_{n-r}
\end{array}\right)\right)
\end{aligned}
$$

if $1 \leq r<n$ and the limit exists, where $V^{\prime}=\left(V_{1}^{\prime}, V_{2}^{\prime}\right) \in \mathbb{C}_{2 r}^{m}$ and $Z^{\prime} \in \mathbb{H}^{r}$; by

$$
F \mid \Phi^{n, 0}=\lim _{\lambda \rightarrow+\infty} F\left(0_{2 n}^{m}, \sqrt{-1} \lambda \cdot 1_{n}\right)
$$

when the limit exists; and by

$$
F \mid \Phi^{n, n}=F .
$$

If $F$ is a theta-function (2.7) of genus $n$ of a pair $(Q, H)$ with $H \in \mathbb{M}(Q)$, then the limits (4.1) and (4.2) exist and equal the theta-function of genus $r$ of the same pair,

$$
\left(\Theta^{n} \mid \Phi^{n, r}\right)\left(V^{\prime}, Z^{\prime} ; H, Q\right)=\Theta^{r}\left(V^{\prime}, Z^{\prime} ; H, Q\right) \quad\left(V^{\prime} \in \mathbb{C}_{2 r}^{m}, Z^{\prime} \in \mathbb{H}^{r}\right)
$$

if $1 \leq r<n$, and $\Theta^{n} \mid \Phi^{n, 0}=1$. Actually, the theta-function (2.7) converges absolutely and uniformly when $V$ and $H$ are in fixed compact sets and $Z$ belongs to a set $\mathbb{H}_{\varepsilon}^{n}$ of the form (2.4) with a fixed $\varepsilon>0$, and so the limit can be taken termwise. For the exponent (3.15) we clearly have

$$
\begin{array}{r}
\lim _{\lambda \rightarrow+\infty} \mathbf{e}\left\{\left(V_{1}^{\prime}, 0_{n-r}^{m}, V_{2}^{\prime}, 0_{n-r}^{m}\right),\left(\begin{array}{cc}
Z^{\prime} & 0_{n-r}^{r} \\
0_{r}^{n-r} & \sqrt{-1} \lambda \cdot 1_{n-r}
\end{array}\right), H, Q ;\left(N_{1}, N_{2}\right)\right\} \\
=\lim _{\lambda \rightarrow+\infty} \mathbf{e}\left\{\sqrt{-1} \lambda \cdot H\left[N_{2}\right]\right\} \mathbf{e}\left\{\left(V_{1}^{\prime}, V_{2}^{\prime}\right), Z^{\prime}, H, Q ; N_{1}\right\} \\
= \begin{cases}\mathbf{e}\left\{\left(V_{1}^{\prime}, V_{2}^{\prime}\right), Z^{\prime}, H, Q ; N_{1}\right\} & \text { if } N_{2}=0_{n-r}^{m}, \\
0 & \text { if } N_{2} \neq 0_{n-r}^{m} .\end{cases}
\end{array}
$$

For $n>r \geq 1$, the Zharkovskaya homomorphism related to the action of Hecke operators on theta-functions of genus $n$ of the quadratic form in $m=2 k$ variables with matrix $Q$,

$$
\Psi^{n, r}=\Psi_{Q}^{n, r}: \mathcal{H}_{0}^{n}(q) \rightarrow \mathcal{H}_{0}^{r}(q),
$$

can be defined as follows. If $T \in \mathcal{H}_{0}^{n}(q)$ is of the form (3.2) with triangular representatives $M_{\alpha}$ of the form (3.9), then after replacing each representative $M_{\alpha}$ by $\left(\begin{array}{cc}{ }^{t} U_{\alpha}^{-1} & 0 \\ 0 & U_{\alpha}\end{array}\right) M_{\alpha}$ with suitable $U_{\alpha} \in \mathrm{GL}_{n}(\mathbb{Z})$ we may assume that the block $D_{\alpha}$ of $M_{\alpha}$ has the form $D_{\alpha}=\left(\begin{array}{cc}D_{\alpha}^{\prime} & * \\ 0 & D_{\alpha}^{\prime \prime}\end{array}\right)$ with $D_{\alpha}^{\prime} \in \mathbb{Z}_{r}^{r}$ (see, e.g., [An87, Lemma 3.2.7]), so that

$$
M_{\alpha}=\left(\begin{array}{cc}
\left(\begin{array}{cc}
A_{\alpha}^{\prime} & 0 \\
* & A_{\alpha}^{\prime \prime}
\end{array}\right) & \left(\begin{array}{cc}
B_{\alpha}^{\prime} & * \\
* & *
\end{array}\right) \\
0 & \left(\begin{array}{cc}
D_{\alpha}^{\prime} & * \\
0 & D_{\alpha}^{\prime \prime}
\end{array}\right)
\end{array}\right)
$$

where 


$$
M_{\alpha}^{\prime}=\left(\begin{array}{cc}
A_{\alpha}^{\prime} & B_{\alpha}^{\prime} \\
0 & D_{\alpha}^{\prime}
\end{array}\right) \in \Sigma_{0}^{r}(q) \quad \text { with } \mu\left(M_{\alpha}^{\prime}\right)=\mu\left(M_{\alpha}\right) .
$$

Then we put

$$
\Psi^{n, r}(T)=\Psi_{Q}^{n, r}(T)=\sum_{\alpha} c_{\alpha} j_{Q}\left(D_{\alpha}^{\prime \prime}\right)^{-1}\left(\Gamma_{0}^{r}(q) M_{\alpha}^{\prime}\right),
$$

where $j_{Q}$ has the form (3.14). It can be easily verified that the linear combination (4.8) belongs to the ring $\mathcal{H}_{0}^{r}(q)$, and that the mapping $T \mapsto \Psi^{n, r}(T)$ is a $\mathbb{C}$-linear homomorphism of rings. For $r=0$ and $r=n$ we set

$$
\Psi^{n, 0}(T)=\Psi_{Q}^{n, 0}(T)=\sum_{\alpha} c_{\alpha} j_{Q}\left(D_{\alpha}\right)^{-1}, \quad \Psi^{n, n}(T)=\Psi_{Q}^{n, n}(T)=T .
$$

It is clear that the Zharkovskaya homomorphism with $r \geq 1$ maps homogeneous elements of $\mathcal{H}_{0}^{n}(q)$ to homogeneous elements of the same multipliers.

Proposition 4.1 (Zharkovskaya commutation relation for theta-functions). Let $Q$ be a nonsingular even matrix of an even order $m=2 k$ with signature $(s, l)$ and level $q, H \in \mathbb{M}(Q)$, and let $T \in \mathcal{H}_{0}^{n}(q)$. Then the following commutation relation holds for the action of the Hecke operator $\left.\right|_{\mathbf{j}} T$ with $\mathbf{j}=j_{Q}(M, Z)$ of the form (2.12) on the theta-function (2.7) of genus $n$ :

$$
\begin{aligned}
\left(\left(\Theta^{n}{ }_{\mathbf{j}} T\right) \mid \Phi^{n, r}\right)\left(V^{\prime}, Z^{\prime} ; H, Q\right) & =\left(\left.\left(\Theta^{n} \mid \Phi^{n, r}\right)\right|_{\mathbf{j}} \Psi^{n, r}(T)\right)\left(V^{\prime}, Z^{\prime} ; H, Q\right) \\
& =\left(\Theta^{r}{ }_{\mathbf{j}} \Psi^{n, r}(T)\right)\left(V^{\prime}, Z^{\prime} ; H, Q\right)
\end{aligned}
$$

if $n>r \geq 1$, where $V^{\prime}=\left(V_{1}^{\prime}, V_{2}^{\prime}\right) \in \mathbb{C}_{2 r}^{m}$, and $Z^{\prime} \in \mathbb{H}^{r}$, and

$$
\left(\left.\Theta^{n}\right|_{\mathbf{j}} T\right) \mid \Phi^{n, 0}=\left(\Theta^{n} \mid \Phi^{n, 0}\right) \cdot \Psi^{n, 0}(T)=\Psi^{n, 0}(T) .
$$

Proof. It suffices to assume that $n>r \geq 1$, since the degenerate case $r=0$ directly follows from conditions (4.2), (4.3), and (4.9). We may assume that $T$ is homogeneous with $\mu(T)=\mu$ and with triangular representatives $M_{\alpha}$ of the form (4.7). By (3.10) with $P=1$ and (4.1), we obtain

$$
\begin{aligned}
\lim _{\lambda \rightarrow+\infty} & \left(\left.\Theta^{n}\right|_{\mathbf{j}} T\right)\left(\widetilde{V}, Z_{\lambda} ; H, Q\right) \\
\quad= & \lim _{\lambda \rightarrow+\infty} \sum_{N \in \mathbb{Z}_{n}^{m}} \sum_{\alpha} c_{\alpha} j_{Q}\left(M_{\alpha}, Z_{\lambda}\right)^{-1} \mathbf{e}\left\{\widetilde{V}^{t} M_{\alpha}, M_{\alpha}\left\langle Z_{\lambda}\right\rangle, H, Q ; N\right\},
\end{aligned}
$$

where

$$
\widetilde{V}=\left(V_{1}^{\prime}, 0_{n-r}^{m}, V_{2}^{\prime}, 0_{n-r}^{m}\right) \quad \text { and } \quad Z_{\lambda}=\left(\begin{array}{cc}
Z^{\prime} & 0_{n-r}^{m} \\
0_{m}^{n-r} & \sqrt{-1} \lambda \cdot 1_{n-r}
\end{array}\right) .
$$

By (3.14) and (3.16), the last expression can be written as

$$
\lim _{\lambda \rightarrow+\infty} \sum_{N \in \mathbb{Z}_{n}^{m}} \sum_{\alpha} c_{\alpha} j_{Q}\left(D_{\alpha}\right)^{-1} \mathbf{e}\left\{B_{\alpha} D_{\alpha}^{-1} Q[N]\right\} \mathbf{e}\left\{\mu \widetilde{V}, Z_{\lambda}, \mu^{-1} H, \mu^{-1} Q ; N A_{\alpha}\right\},
$$

where $\mu=\mu(T)=\mu\left(M_{\alpha}\right)$. According to (4.5), this limit is equal to 


$$
\begin{aligned}
& \sum_{N \in \mathbb{Z}_{n}^{m}} \sum_{\alpha} c_{\alpha} j_{Q}\left(D_{\alpha}\right)^{-1} \mathbf{e}\left\{B_{\alpha} D_{\alpha}^{-1} Q[N]\right\} \\
& \times \lim _{\lambda \rightarrow+\infty} \mathbf{e}\left\{\mu \widetilde{V}, Z_{\lambda}, \mu^{-1} H, \mu^{-1} Q ; N A_{\alpha}\right\} \\
&=\sum_{N_{1} \in \mathbb{Z}_{r}^{m}} \sum_{\alpha} c_{\alpha} j_{Q}\left(D_{\alpha}\right)^{-1} \mathbf{e}\left\{B_{\alpha} D_{\alpha}^{-1} Q\left[N_{1}, 0_{n-r}^{m}\right]\right\} \\
& \times \mathbf{e}\left\{\mu\left(V_{1}^{\prime}, V_{2}^{\prime}\right), Z^{\prime}, \mu^{-1} H, \mu^{-1} Q ; N_{1} A_{\alpha}^{\prime}\right\} .
\end{aligned}
$$

By the obvious relations

$$
\mathbf{e}\left\{B_{\alpha} D_{\alpha}^{-1} Q\left[N_{1}, 0_{n-r}^{m}\right]\right\}=\mathbf{e}\left\{B_{\alpha}^{\prime}\left(D_{\alpha}^{\prime}\right)^{-1} Q\left[N_{1}\right]\right\},
$$

and formulas (3.16) with $M=M_{\alpha}^{\prime}$, we arrive at the sum

$$
\begin{aligned}
\sum_{N_{1} \in \mathbb{Z}_{r}^{m}} \sum_{\alpha} c_{\alpha} j_{Q}\left(D_{\alpha}^{\prime} D_{\alpha}^{\prime \prime}\right)^{-1} \mathbf{e}\left\{\left(V_{1}^{\prime}, V_{2}^{\prime}\right)^{t} M_{\alpha}^{\prime}, M_{\alpha}^{\prime}\left\langle Z^{\prime}\right\rangle, H, Q ; N_{1}\right\} \\
=\left.\sum_{\alpha} c_{\alpha} j_{Q}\left(D_{\alpha}^{\prime \prime}\right)^{-1} \Theta^{r}\left(\left(V_{1}^{\prime}, V_{2}^{\prime}\right), Z^{\prime} ; H, Q\right)\right|_{\mathbf{j}} M_{\alpha}^{\prime} \\
=\left(\Theta^{r} \mid\left(\Psi^{n, r}(T)\right)\left(\left(V_{1}^{\prime}, V_{2}^{\prime}\right), Z^{\prime} ; H, Q\right) .\right.
\end{aligned}
$$

The Zharkovskaya homomorphism (4.4) is not always epimorphic (see, e.g., [An96, Proposition 3.3]).

The following useful lemma is an elementary consequence of definitions.

Lemma 4.2. If $T \in \mathcal{H}_{0}^{n}(q)$ and $N \in C^{r}(Q / \mu)$, where $n>r>0$, then

$$
I\left(\left(N, 0_{n-r}^{m}\right), Q, T\right)=I\left(N, Q, \Psi^{n, r}(T)\right),
$$

where $\Psi^{n, r}=\Psi_{Q}^{n, r}$ is the Zharkovskaya homomorphism; moreover,

$$
I\left(0_{n}^{m}, Q, T\right)=\Psi^{n, 0}(T) .
$$

Proof. In order to prove (4.12) we may assume that $T$ is a homogeneous element of the form (3.2) with triangular representatives $M_{\alpha}$ of the form (4.7), where $\mu\left(M_{\alpha}\right)=\mu(T)=\mu$. By (3.13) we have

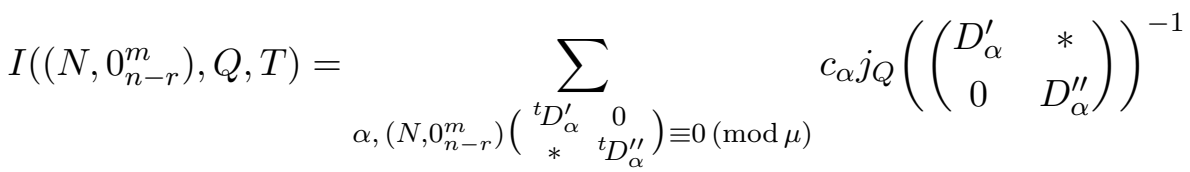

$$
\begin{aligned}
& \times \mathbf{e}\left\{\mu^{-2}\left(\begin{array}{cc}
Q[N] & 0 \\
0 & 0
\end{array}\right) \cdot\left(\begin{array}{cc}
{ }^{t} D_{\alpha}^{\prime} B_{\alpha}^{\prime} & * \\
& *
\end{array}\right)\right\} \\
& =\sum_{\alpha, N^{t} D_{\alpha}^{\prime} \equiv 0(\bmod \mu)} c_{\alpha} j_{Q}\left(D_{\alpha}^{\prime \prime}\right)^{-1} j_{Q}\left(D_{\alpha}^{\prime}\right)^{-1} \mathbf{e}\left\{\mu^{-2} Q[N]^{t} D_{\alpha}^{\prime} B_{\alpha}^{\prime}\right\} \\
& =I\left(N, Q, \Psi^{n, r}(T)\right) .
\end{aligned}
$$

Formula (4.13) directly follows from (3.13) and (4.9). 
5. Automorphic expansion of interaction sums and explicit formulas. We are going to investigate here the role played by iSums in deduction of finite formulas for the action of Hecke operators on theta-functions. Since these sums do not depend on harmonic factors in the definition of theta-functions, for their investigation one can use theta-functions (2.7) without harmonic factors.

In this section we again assume that $Q$ is the matrix of a fixed integral nonsingular quadratic form $\mathbf{q}(X)$ in an even number $m=2 k$ of variables, $(s, l)$ is the signature of $\mathbf{q}, q$ the level of $Q, \chi_{Q}$ the corresponding Dirichlet character modulo $q$, and $H \in \mathbb{M}(Q)$.

We shall say that an integral $m \times m$-matrix $D$ is an automorph of the matrix $Q$ (or an automorph of the form $\mathbf{q}$ ) with multiplier $\mu=\mu(D)$ if

$$
\mu^{-1} Q[D] \in \mathbb{E}^{m} \text { and } \operatorname{det} \mu^{-1} Q[D]=\operatorname{det} Q .
$$

The set of all automorphs of $Q$ with multiplier $\mu$ will be denoted by

$$
A(Q, \mu)=\left\{D \in \mathbb{Z}_{m}^{m} \mid \mu^{-1} Q[D] \in \mathbb{E}^{m}, \operatorname{det} \mu^{-1} Q[D]=\operatorname{det} Q\right\} .
$$

The set $A(Q, \mu)$ may be empty (see, for example, Proposition 6.2 below). It is clear that

$$
A(Q, \mu) \Lambda^{m}=A(Q, \mu), \quad \text { where } \quad \Lambda^{m}=\mathrm{GL}_{m}(\mathbb{Z}),
$$

and so the group $\Lambda^{m}$ operates on each of the sets $A(Q, \mu)$ by right multiplication. Since all automorphs of $A(Q, \mu)$ are integral matrices of determinants $\pm \mu^{m / 2}$, it follows that each set of classes of automorphs $A(Q, \mu) / \Lambda^{m}$ is finite.

Proposition 5.1. Let $T$ be a homogeneous nonzero element of $\mathcal{H}_{0}^{m}(q)$ with multiplier $\mu(T)=\mu$. Then the following three conditions are equivalent.

(1) The action of the Hecke operator $\left.\right|_{\mathbf{j}} T$ with $\mathbf{j}=j_{Q}(M, Z)$ on the thetafunction (2.7) of genus $n=m$ is given by

$$
\begin{array}{r}
\left.\Theta^{m}(V, Z ; H, Q)\right|_{\mathbf{j}} T \\
\sum_{D \in A(Q, \mu) / \Lambda} c(D, Q, T) \Theta^{m}\left(\mu D^{-1} V, Z ; \mu^{-1} H[D], \mu^{-1} Q[D]\right) \\
0
\end{array}
$$

where $\Lambda=\Lambda^{m}$, with some coefficients $c(D, Q, T)$ depending only on $D, Q$, and $T$.

(2) The formula (5.2) is valid with the coefficients of the form

$$
c(D, Q, T)=I(D, Q, T),
$$

where $I(D, Q, T)$ are the iSums (3.13).

(3) (The automorphic expansion of iSums) For each matrix $N \in C^{m}(Q / \mu)$ in the set (3.12) with $n=m$ the following formulas hold: 


$$
I(N, Q, T)= \begin{cases}\sum_{D \in A(Q, \mu) / \Lambda, D \mid N} I(D, Q, T) & \text { if } A(Q, \mu) \neq \emptyset, \\ 0 & \text { if } A(Q, \mu)=\emptyset,\end{cases}
$$

where the condition $D \mid N$ means that the matrix $D^{-1} N$ is integral.

Proof. By (3.11) with $n=m$ and $P=1$, we can rewrite the left hand side of (5.2) in the case $A(Q, \mu) \neq \emptyset$ in the form

$$
\begin{array}{r}
\sum_{N \in C^{m}(Q / \mu)} I(N, Q, T) \mathbf{e}\left\{\mu^{-1} Q\left[N-\mu V_{2}\right] X+\sqrt{-1} \mu^{-1} H\left[N-\mu V_{2}\right] Y\right. \\
\left.+2^{t}\left(\mu V_{1}\right) \mu^{-1} Q N-{ }^{t}\left(\mu V_{1}\right) \mu^{-1} Q\left(\mu V_{2}\right)\right\} \\
=\sum_{D \in A(Q, \mu) / \Lambda} c(D, Q, T) \Theta^{m}\left(\mu D^{-1} V, Z ; \mu^{-1} H[D], \mu^{-1} Q[D]\right) .
\end{array}
$$

By setting here $V_{2}=0_{m}^{m}$, we get the relation

$$
\begin{array}{r}
\sum_{N \in C^{m}(Q / \mu)} I(N, Q, T) \mathbf{e}\left\{\mu^{-1} Q[N] X+\sqrt{-1} \mu^{-1} H[N] Y+2{ }^{t} V_{1} Q N\right\} \\
=\sum_{D \in A(Q, \mu) / \Lambda} c(D, Q, T) \sum_{N \in \mathbb{Z}_{m}^{m}} \mathbf{e}\left\{\mu^{-1} Q[D N] X+\sqrt{-1} \mu^{-1} H[D N] Y\right. \\
\left.+2^{t}\left(\mu D^{-1} V_{1}\right) \mu^{-1}{ }^{t} D Q D N\right\} .
\end{array}
$$

Collecting in the last sum the terms with $D N=\widetilde{N} \in \mathbb{Z}_{m}^{m}$ we can rewrite it in the form

$$
\sum_{\widetilde{N} \in \mathbb{Z}_{m}^{m}}\left(\sum_{\substack{D \in A(Q, \mu) / \Lambda \\ D^{-1} \widetilde{N} \in \mathbb{Z}_{m}^{m}}} c(D, Q, T) \mathbf{e}\left\{\mu^{-1} Q[\widetilde{N}] X+\sqrt{-1} \mu^{-1} H[\tilde{N}] Y\right\}\right) \mathbf{e}\left\{2{ }^{t} V_{1} Q \widetilde{N}\right\}
$$

If $\widetilde{N} \in A(Q, \mu) / \Lambda$, then the condition $D^{-1} \widetilde{N} \in \mathbb{Z}_{m}^{m}$ with $D \in A(Q, \mu) / \Lambda$ implies $\widetilde{N} \Lambda=D \Lambda$, i.e., $\widetilde{N}=D$. Therefore, the coefficient of $\mathbf{e}\left\{2{ }^{t} V_{1} Q D\right\}$ in the last sum is equal to

$$
c(D, Q, T) \mathbf{e}\left\{\mu^{-1} Q[D] X+\sqrt{-1} \mu^{-1} H[D] Y\right\} .
$$

Comparing it with the corresponding coefficient on the left, we get (5.3).

From (3.11) and formula (5.2) for the case $A(Q, \mu) \neq \emptyset$ with coefficients of the form (5.3) we get the identity

$$
\begin{aligned}
\sum_{N \in C^{m}(Q / \mu)} I(N, Q, T) \mathbf{e}\left\{\mu^{-1} Q\left[N-\mu V_{2}\right] X+\sqrt{-1} \mu^{-1} H\left[N-\mu V_{2}\right] Y\right. \\
\left.+2^{t}\left(\mu V_{1}\right) \mu^{-1} Q N-{ }^{t}\left(\mu V_{1}\right) \mu^{-1} Q\left(\mu V_{2}\right)\right\}
\end{aligned}
$$




$$
\begin{aligned}
& =\sum_{D \in A(Q, \mu) / \Lambda} I(D, Q, T) \Theta^{m}\left(\mu D^{-1} V, Z ; \mu^{-1} H[D], \mu^{-1} Q[D]\right) \\
& =\sum_{D \in A(Q, \mu) / \Lambda} I(D, Q, T) \sum_{N^{\prime} \in \mathbb{Z}_{m}^{m}} \mathbf{e}\left\{\mu^{-1} Q[D]\left[N^{\prime}-\mu D^{-1} V_{2}\right] X\right. \\
& +\sqrt{-1} \mu^{-1} H[D]\left[N^{\prime}-\mu D^{-1} V_{2}\right] Y \\
& \left.+2^{t}\left(\mu V_{1}\right) \mu^{-1} Q D N^{\prime}-{ }^{t}\left(\mu V_{1}\right) \mu^{-1} Q\left(\mu V_{2}\right)\right\} \\
& =\sum_{N \in C^{m}(Q / \mu)}\left(\sum_{D \in A(Q, \mu) / \Lambda, D \mid N} I(D, Q, T)\right) \mathbf{e}\left\{\mu^{-1} Q\left[N-\mu V_{2}\right] X\right. \\
& \left.+\sqrt{-1} \mu^{-1} H\left[N-\mu V_{2}\right] Y+2^{t}\left(\mu V_{1}\right) \mu^{-1} Q N-{ }^{t}\left(\mu V_{1}\right) \mu^{-1} Q\left(\mu V_{2}\right)\right\},
\end{aligned}
$$

because clearly $N=D N^{\prime} \in C^{m}(Q / \mu)$ if $D \in A(Q, \mu)$ and $N^{\prime} \in \mathbb{Z}_{m}^{m}$. By putting here $V_{2}=0$ and comparing the $N$ th Fourier coefficients of the first and last series, by the uniqueness of the Fourier expansion of the analytical and periodic (in $V_{1}$ ) function $\left(\Theta^{m} \mid \mathbf{j} T\right)\left(\left(V_{1}, 0\right), Z ; H, Q\right)$, we obtain the first case of (5.4). If $A(Q, \mu)=\emptyset$, then it is easy to see that the second case of (5.2) and (3.11) with $n=m$ and $P=1$ imply the second case of (5.4).

Finally, if (3) is true, then in the case $A(Q, \mu) \neq \emptyset$, by (3.11) with $n=m$ and $P=1$ and (5.4), we obtain

$$
\begin{aligned}
& \left.\Theta^{m}(V, Z ; H, Q)\right|_{\mathbf{j}} T=\sum_{N \in C^{m}(Q / \mu)}\left(\sum_{D \in A(Q, \mu) / \Lambda, D \mid N} I(D, Q, T)\right) \\
& \times \mathbf{e}\left\{\mu^{-1} Q\left[N-\mu V_{2}\right] X+\sqrt{-1} \mu^{-1} H\left[N-\mu V_{2}\right] Y\right. \\
& \left.+2^{t}\left(\mu V_{1}\right) \mu^{-1} Q N-{ }^{t}\left(\mu V_{1}\right) \mu^{-1} Q\left(\mu V_{2}\right)\right\} \\
& =\sum_{D \in A(Q, \mu) / \Lambda} I(D, Q, T) \sum_{N=D N^{\prime}, N^{\prime} \in \mathbb{Z}_{m}^{m}} \mathbf{e}\left\{\mu^{-1} Q\left[N-\mu V_{2}\right] X\right. \\
& \left.+\sqrt{-1} \mu^{-1} H\left[N-\mu V_{2}\right] Y+2^{t}\left(\mu V_{1}\right) \mu^{-1} Q N-{ }^{t}\left(\mu V_{1}\right) \mu^{-1} Q\left(\mu V_{2}\right)\right\} \\
& =\sum_{D \in A(Q, \mu) / \Lambda} I(D, Q, T) \sum_{N^{\prime} \in \mathbb{Z}_{m}^{m}} \mathbf{e}\left\{\mu^{-1} Q[D]\left[N^{\prime}-\mu D^{-1} V_{2}\right] X\right. \\
& +\sqrt{-1} \mu^{-1} H[D]\left[N^{\prime}-\mu D^{-1} V_{2}\right] Y \\
& \left.+2^{t}\left(\mu V_{1}\right) \mu^{-1} Q D N^{\prime}-{ }^{t}\left(\mu V_{1}\right) \mu^{-1} Q\left(\mu V_{2}\right)\right\} \\
& =\sum_{D \in A(Q, \mu) / \Lambda} I(D, Q, T) \Theta^{m}\left(\mu D^{-1} V, Z ; \mu^{-1} H[D], \mu^{-1} Q[D]\right) \text {. }
\end{aligned}
$$

But if $A(Q, \mu)=\emptyset$, the second case of (5.4) and formula (3.11) imply the second case of (5.2).

Let us now turn to an arbitrary genus $n \geq 1$.

Proposition 5.2. Let $T$ be a homogeneous element of $\mathcal{H}_{0}^{n}(q)$ with multiplier $\mu(T)=\mu$, satisfying the condition 


$$
T \in \Psi_{Q}^{m, n}\left(\mathcal{H}_{0}^{m}(q)\right) \text { if } n<m,
$$

where $\Psi_{Q}^{m, n}$ is the Zharkovskaya map (4.6). Suppose that the element $\Psi^{n, m} T$ $\in \mathcal{H}_{0}^{m}(q)$, where, for $n<m, \Psi_{Q}^{n, m} T$ is an inverse image of $T$ under the map $\Psi_{Q}^{m, n}$, satisfies at least one of the equivalent conditions (1), (2), or (3) of Proposition 5.1. Then the following two assertions hold:

(1) (Automorphic expansions of iSums of genus $n$ ) For each matrix $N \in$ $C^{n}(Q / \mu)$ the following expansions of the iSums hold:

$$
I(N, Q, T)= \begin{cases}\sum_{D \in A(Q, \mu) / \Lambda, D \mid N} I\left(D, Q, \Psi_{Q}^{n, m} T\right) & \text { if } A(Q, \mu) \neq \emptyset, \\ 0 & \text { if } A(Q, \mu)=\emptyset,\end{cases}
$$

where $\Lambda=\Lambda^{m}=\mathrm{GL}_{m}(\mathbb{Z})$ and the condition $D \mid N$ means that the matrix $D^{-1} N$ is integral.

(2) (Explicit formulas for the action of $\left.\right|_{\mathbf{j}} T$ on a theta-function of genus $n$ with a harmonic form) For each polynomial harmonic form $P$ on $\mathbb{C}_{n}^{m}$ relative to the pair $(Q, H)$ satisfying $(2.9)$,

$$
=\left\{\begin{array}{l}
\sum_{D \in A(Q, \mu) / \Lambda} I\left(D, Q, \Psi_{Q}^{n, m} T\right) \Theta_{P \mid \mu^{-1} D}^{n}\left(\mu D^{-1} V, Z ; \mu^{-1} H[D], \mu^{-1} Q[D]\right), \\
0,
\end{array}\right.
$$

depending on whether $A(Q, \mu) \neq \emptyset$ or $A(Q, \mu)=\emptyset$, where $\mathbf{j}=j_{Q, P}(M, Z)$ is the automorphic factor (2.14), and

$$
\left(P \mid \mu^{-1} D\right)(X)=P\left(\mu^{-1} D X\right) \quad\left(X \in \mathbb{C}_{n}^{m}\right) .
$$

Proof. If $n>m$ and $N \in C^{n}(Q / \mu)$, then it is easy to see that there is a matrix $\gamma \in \mathrm{GL}_{n}(\mathbb{Z})$ such that $N \gamma=\left(N^{\prime}, 0_{n-m}^{m}\right)$ with $N^{\prime} \in C^{m}(Q / \mu)$. By Lemmas 3.2 and 4.2 , we obtain

$$
I(N, Q, T)=I\left(\left(N^{\prime}, 0_{n-m}^{m}\right), Q, T\right)=I\left(N^{\prime}, Q, \Psi^{n, m} T\right),
$$

and, by (5.4) for the case $A(Q, \mu) \neq \emptyset$, we have

$$
\begin{aligned}
I\left(N^{\prime}, Q, \Psi^{n, m} T\right) & =\sum_{D \in A(Q, \mu) / \Lambda^{m}, D \mid N^{\prime}} I\left(D, Q, \Psi^{n, m} T\right) \\
& =\sum_{D \in A(Q, \mu) / \Lambda^{m}, D \mid N} I\left(D, Q, \Psi^{n, m} T\right),
\end{aligned}
$$

since the conditions $D \mid N^{\prime}$ and $D \mid N$ are clearly equivalent, which proves the first case of (5.6). But if $n \leq m$ and $N \in C^{n}(Q / \mu)$, then clearly $N^{\prime}=$ $\left(N, 0_{m-n}^{m}\right) \in C^{m}(Q / \mu)$ and, by $(5.5), \Psi_{Q}^{n, m} T \in \mathcal{H}_{0}^{m}(q)$. Hence, by the first 
case of (5.4), we can write

$$
\begin{aligned}
I\left(N^{\prime}, Q, \Psi_{Q}^{n, m} T\right) & =\sum_{D \in A(Q, \mu) / \Lambda^{m}, D \mid N^{\prime}} I\left(D, Q, \Psi_{Q}^{n, m}(T)\right) \\
& =\sum_{D \in A(Q, \mu) / \Lambda^{m}, D \mid N} I\left(D, Q, \Psi_{Q}^{n, m} T\right) .
\end{aligned}
$$

On the other hand, by (4.12),

$$
I\left(N^{\prime}, Q, \Psi^{n, m} T\right)=I\left(N, Q, \Psi^{m, n}\left(\Psi^{n, m} T\right)\right)=I(N, Q, T) .
$$

These arguments prove part (1) if $A(Q, \mu) \neq \emptyset$. But if $A(Q, \mu)=\emptyset$, then by (5.8) and the second case of (5.4) we see that $I(N, Q, T)=0$ for all $N \in C^{n}(Q, \mu)$ if $n>m$. But if $n \leq m$ and $N \in C^{n}(Q / \mu)$, then again $N^{\prime}=\left(N, 0_{m-n}^{m}\right) \in C^{m}(Q / \mu)$ and, by $(5.5), \Psi_{Q}^{n, m} T \in \mathcal{H}_{0}^{m}(q)$. By the second case of (5.4) we have $I\left(N^{\prime}, Q, \Psi_{Q}^{n, m} T\right)=0$, which, by (5.9), implies the second case of (5.6).

Part (2) follows from (1) and Proposition 3.1. Indeed, from (3.11) and the first case of (5.6) we get the formula

$$
\begin{aligned}
& \left.\Theta_{P}^{n}\left(\left(V_{1}, V_{2}\right), X+\sqrt{-1} Y ; H, Q\right)\right|_{\mathbf{j}} T \\
& =\sum_{N \in C^{n}(Q / \mu)}\left(\sum_{D \in A(Q, \mu) / \Lambda, D \mid N} I\left(D, Q, \Psi^{n, m} T\right)\right) P\left(\mu^{-1}\left(N-\mu V_{2}\right)\right) \\
& \times \mathbf{e}\left\{\mu^{-1} Q\left[N-\mu V_{2}\right] X+\sqrt{-1} \mu^{-1} H\left[N-\mu V_{2}\right] Y\right. \\
& \left.+2^{t}\left(\mu V_{1}\right) \mu^{-1} Q N-{ }^{t}\left(\mu V_{1}\right) \mu^{-1} Q\left(\mu V_{2}\right)\right\} \\
& =\sum_{D \in A(Q, \mu) / \Lambda} I\left(D, Q, \Psi^{n, m} T\right) \sum_{N=D N^{\prime}, N^{\prime} \in \mathbb{Z}_{n}^{m}} P\left(\mu^{-1}\left(N-\mu V_{2}\right)\right) \\
& \times \mathbf{e}\left\{\mu^{-1} Q\left[N-\mu V_{2}\right] X+\sqrt{-1} \mu^{-1} H\left[N-\mu V_{2}\right] Y\right. \\
& \left.+2^{t}\left(\mu V_{1}\right) \mu^{-1} Q N-{ }^{t}\left(\mu V_{1}\right) \mu^{-1} Q\left(\mu V_{2}\right)\right\} \\
& =\sum_{D \in A(Q, \mu) / \Lambda} I\left(D, Q, \Psi^{n, m} T\right) \sum_{N^{\prime} \in \mathbb{Z}_{n}^{m}} P\left(\mu^{-1} D\left(N^{\prime}-\mu D^{-1} V_{2}\right)\right) \\
& \times \mathbf{e}\left\{\mu^{-1} Q[D]\left[N^{\prime}-\mu D^{-1} V_{2}\right] X+\sqrt{-1} \mu^{-1} H[D]\left[N^{\prime}-\mu D^{-1} V_{2}\right] Y\right. \\
& \left.+2^{t}\left(\mu V_{1}\right) \mu^{-1} Q D N^{\prime}-{ }^{t}\left(\mu V_{1}\right) \mu^{-1} Q\left(\mu V_{2}\right)\right\} \\
& =\sum_{D \in A(Q, \mu) / \Lambda} I\left(D, Q, \Psi^{n, m} T\right) \Theta_{P \mid \mu^{-1} D}^{n}\left(\mu D^{-1} V, Z ; \mu^{-1} H[D], \mu^{-1} Q[D]\right) \text {, }
\end{aligned}
$$

which proves the first case of (5.7). The second directly follows from the second case of (5.6) and formula (3.11).

In this section we have deduced several reformulations of transformation formulas for the action of Hecke operators on theta-functions by using quite formal computations. However, for real proof of certain basic formulas 
one cannot escape arithmetical considerations. In our work [An86, Theorem 1] formulas (5.2) were completely proved, although, for accuracy, it should be noted that, as follows from the proofs of that theorem and related lemmas, their formulations must be completed by the statement that all sums over classes of automorphs are set to be zero when the corresponding automorphs do not exist, just as above in the formulations of Propositions 5.1 and 5.2. The proof of the formulas is heavily based on a complicated technique of explicit factorizations of certain standard polynomials (Rankin polynomials) over symplectic Hecke-Shimura rings under their parabolic embeddings, which was earlier developed in [An79, Chapter 2] and later detailed in $[$ An87, §3.5]. Perhaps, it could be useful to write now a simplified exposition of the proof, but it would require plenty of time without any new method or results at the end. As we have seen above, formulas (5.2) imply (5.3), (5.4), (5.6), and (5.7). On the other hand, in order to justify all these formulas it would be sufficient to prove independently the elementary formulas (5.4) for fairly simple trigonometric sums $I(N, Q, T)$, which, in general, has not been done yet. In the rest of this paper we consider some cases where the formulas can be proved elementarily without using parabolic embeddings.

6. Action of Hecke operators corresponding to $T^{n}(p)$. In this section we preserve the assumptions and notation of the previous section. In particular, $Q$ is the matrix of a fixed integral nonsingular quadratic form $\mathbf{q}(X)$ in an even number $m=2 k$ of variables, $q$ the level of $Q, \chi_{Q}$ the corresponding Dirichlet character modulo $q$, and $H \in \mathbb{M}(Q)$. By using an elementary approach, we shall prove the formulas (5.7) for the action of the Hecke operators $\left.\right|_{\mathbf{j}} T^{n}(p)$ with $p$ prime not dividing $q$ on theta-functions (2.8) of genus $n$ with harmonic coefficients. Similar formulas for theta-series (2.2) of positive definite quadratic forms (without harmonic coefficients) were proved in [An80] and by another method in [An87, §5.2.2]. Here we generalize the approach used in [An80]. According to the approach of the previous section, we consider first the case when the harmonic form $P$ equals 1 . We start with direct formulas for the action of the operator corresponding to $T^{n}(p)$ on the theta-function (2.7).

Proposition 6.1. For each prime $p$ not dividing the level $q$ of $Q$, for every $n=1,2, \ldots$ and for each $N \in C^{n}(Q / p)$ the corresponding iSum can be written in the form

$$
\begin{aligned}
& I\left(N, Q, T^{n}(p)\right) \\
& \quad=\chi_{Q}\left(p^{r(N)}\right) p^{-r(N) k+r(N)(r(N)+1) / 2} \prod_{\gamma=1}^{n-r(N)}\left(1+\chi_{Q}(p) p^{\gamma+r(N)-k}\right),
\end{aligned}
$$


where $r(N)=r_{p}(N)$ is the rank of $N$ over the field $\mathbb{F}_{p}=\mathbb{Z} / p \mathbb{Z}$; the action of $\left.\right|_{\mathbf{j}} T^{n}(p)$ with $\mathbf{j}=j_{Q}(M, Z)$ on $\Theta^{n}(V, Z ; H, Q)$ is given in the same notation by the formula

$$
\left.\Theta^{n}(V, Z ; H, Q)\right|_{\mathbf{j}} T^{n}(p)
$$

$$
\begin{aligned}
=\sum_{N \in C^{n}(Q / p)}\left(\chi_{Q}\left(p^{r(N)}\right) p^{-r(N) k+r(N)(r(N)+1) / 2}\right. & \left.\prod_{\gamma=1}^{n-r(N)}\left(1+\chi_{Q}(p) p^{\gamma+r(N)-k}\right)\right) \\
& \times \mathbf{e}\left\{p V, Z, p^{-1} H, p^{-1} Q ; N\right\},
\end{aligned}
$$

where the notation (3.15) is utilized.

Proof. It is well known (see, e.g., [An87, §3.3]) that a decomposition (3.2) of $T^{n}(p)$ with $p$ prime not dividing $q$ into left cosets modulo the group $\Gamma_{0}^{n}(q)$ can be taken in the form

$$
T^{n}(p)=\sum_{\alpha=0}^{n} \sum_{\substack{D \in \Lambda^{n} \backslash \Lambda^{n} D_{\alpha}^{n} \Lambda^{n} \\
B \in B(D) / \equiv(\bmod D)}}\left(\Gamma_{0}^{n}(q)\left(\begin{array}{cc}
p \cdot{ }^{t} D^{-1} & B \\
0 & D
\end{array}\right)\right),
$$

where $\Lambda^{n}=\mathrm{GL}_{n}(\mathbb{Z}), D_{\alpha}^{n}=D_{\alpha}^{n}(p)=\left(\begin{array}{cc}1_{n-\alpha} & 0 \\ 0 & p \cdot 1_{\alpha}\end{array}\right), B$ ranges over a system of representatives for the set

$$
B(D)=\left\{B \in \mathbb{Z}_{n}^{n} \mid{ }^{t} B D={ }^{t} D B\right\}
$$

modulo the equivalence relation

$$
B \equiv B^{\prime}(\bmod D) \Leftrightarrow\left(B-B^{\prime}\right) D^{-1} \in \mathbb{Z}_{n}^{n} .
$$

Moreover, a system of representatives for $\Lambda^{n} \backslash \Lambda^{n} D_{\alpha}^{n} \Lambda^{n}$ can be taken in the form

$$
\begin{aligned}
& \Lambda^{n} \backslash \Lambda^{n} D_{\alpha}^{n} \Lambda^{n} \\
& \quad=\left\{D_{\alpha}^{n} \varepsilon(\mathbf{x}) \mid \mathbf{x} \in P_{n-\alpha, n}(p) \text { with } \varepsilon(\mathbf{x})=\left(\begin{array}{c}
\mathbf{x} \\
\widehat{\mathbf{x}}
\end{array}\right) \in \mathrm{SL}_{n}(\mathbb{Z})\right\},
\end{aligned}
$$

where $P_{n-\alpha, n}(p)$ is a system for representatives for the set of all matrices $\mathbf{x} \in \mathbb{Z}_{n}^{n-\alpha}$ which can be completed to a matrix $(\underset{\widehat{\mathbf{x}}}{\mathbf{x}}) \in \mathrm{SL}_{n}(\mathbb{Z})$ modulo the equivalence relation

$$
\mathbf{x} \simeq \mathbf{x}^{\prime}(\bmod p) \Leftrightarrow \mathbf{x} \equiv C \mathbf{x}^{\prime}(\bmod p) \text { with } C \in \mathrm{GL}_{n-\alpha}(\mathbb{Z} / p \mathbb{Z}) .
$$

Note that the rank of every $\mathbf{x} \in P_{n-\alpha, n}(p)$ over $\mathbb{F}_{p}$ is $n-\alpha$. 
By using the decomposition (6.3) and formulas (3.13), we have

$$
\begin{aligned}
& I\left(N, Q, T^{n}(p)\right) \\
& =\sum_{\alpha=0}^{n} \sum_{\substack{D \in \Lambda^{n} \backslash \Lambda^{n} D_{\alpha}^{n} \Lambda^{n}, N^{t} D \equiv 0(\bmod p) \\
B \in B(D) / \equiv(\bmod D)}} j_{Q}(D)^{-1} \mathbf{e}\left\{p^{-2} Q[N]^{t} D B\right\} \\
& \quad=\sum_{\alpha=0}^{m} \chi_{Q}\left(p^{\alpha}\right) p^{-\alpha k} \sum_{\substack{D \in \Lambda^{n} \backslash \Lambda^{n} D_{\alpha}^{n} \Lambda^{n}, N^{t} D \equiv 0(\bmod p) \\
B \in B(D) / \equiv(\bmod D)}} \mathbf{e}\left\{p^{-2} B Q[N]^{t} D\right\}
\end{aligned}
$$

since the character $\chi_{Q}=\chi_{Q}^{-1}$ is real. If we take the system of representatives for $\Lambda^{n} \backslash \Lambda^{n} D_{\alpha}^{n} \Lambda^{n}$ in the form (6.4), then, since $B\left(D_{\alpha}^{n} \varepsilon(\mathbf{x})\right)=B\left(D_{\alpha}^{n}\right) \varepsilon(\mathbf{x})$, it follows that a system of representatives for $B\left(D_{\alpha}^{n} \varepsilon(\mathbf{x})\right) / \equiv\left(\bmod D_{\alpha}^{n} \varepsilon(\mathbf{x})\right)$ can be taken in the form

$$
\left\{B\left(D_{\alpha}^{n}\right) / \equiv\left(\bmod D_{\alpha}^{n}\right)\right\} \varepsilon(\mathbf{x})=\left\{B=\left(\begin{array}{cc}
0 & 0 \\
0 & B^{\prime}
\end{array}\right) \mid B^{\prime}={ }^{t} B^{\prime} \in \mathbb{Z}_{\alpha}^{\alpha} / p \mathbb{Z}_{\alpha}^{\alpha}\right\} \varepsilon(\mathbf{x}) .
$$

For $D=D_{\alpha}^{n} \varepsilon(\mathbf{x})=(\underset{p \widehat{\mathbf{x}}}{\mathbf{x}})$ in the set $(6.4)$, the condition $N^{t} D \equiv 0(\bmod p)$ is clearly equivalent to the congruence $N^{t} \mathbf{x} \equiv 0(\bmod p)$. Thus, with these systems of representatives we find that the last sum is equal to

$$
\begin{aligned}
\sum_{\alpha=0}^{n} \chi_{Q}\left(p^{\alpha}\right) p^{-\alpha k} & \\
\times & \sum_{\substack{\mathbf{x} \in P_{n-\alpha, n}(p), N^{t} \mathbf{x} \equiv 0(\bmod p) \\
B^{\prime}={ }^{\prime} B^{\prime} \in \mathbb{Z}_{\alpha}^{\alpha} / p \mathbb{Z}_{\alpha}^{\alpha}}} \mathbf{e}\left\{p^{-2}\left(\begin{array}{cc}
0 & 0 \\
0 & B^{\prime}
\end{array}\right) \varepsilon(\mathbf{x}) Q[N]^{t}\left(D_{\alpha}^{n} \varepsilon(\mathbf{x})\right)\right\} \\
& =\sum_{\alpha=0}^{n} \chi_{Q}\left(p^{\alpha}\right) p^{-\alpha k} \sum_{\substack{\mathbf{x} \in P_{n-\alpha, n}(p), N^{t} \mathbf{x} \equiv 0(\bmod p) \\
B^{\prime}={ }^{t} B^{\prime} \in \mathbb{Z}_{\alpha}^{\alpha} / p \mathbb{Z}_{\alpha}^{\alpha}}} \mathbf{e}\left\{B^{\prime} \widehat{\mathbf{x}} Q[N]^{t} \widehat{\mathbf{x}} / p\right\} \\
& =\sum_{\alpha=0}^{n} \chi_{Q}\left(p^{\alpha}\right) p^{-\alpha k} \sum_{\mathbf{x} \in P_{n-\alpha, n}(p), N^{t} \mathbf{x} \equiv 0(\bmod p)} p^{\alpha(\alpha+1) / 2} \\
& =\sum_{\alpha=0}^{n} \chi_{Q}\left(p^{\alpha}\right) p^{-\alpha k+\alpha(\alpha+1) / 2} R_{\alpha}^{n}(N),
\end{aligned}
$$

where

$$
R_{\alpha}^{n}(N)=\sum_{\mathbf{x} \in P_{n-\alpha, n}(p), N^{t} \mathbf{x} \equiv 0(\bmod p)} 1
$$

since each of the matrices $\widehat{\mathbf{x}} Q[N]^{t} \widehat{\mathbf{x}} / p$, together with the matrix $Q[N] / p$, is even, and the number of symmetric matrices $B^{\prime}$ in $\mathbb{Z}_{\alpha}^{\alpha} / p \mathbb{Z}_{\alpha}^{\alpha}$ is $p^{\alpha(\alpha+1) / 2}$. It is 
clear that $R_{\alpha}^{n}(A N B)=R_{\alpha}^{n}(N)$ if $A, B$ are integral matrices of order $m$ and $n$, respectively, with determinants prime to $p$. Therefore $R_{\alpha}^{n}(N)=R_{\alpha}^{n}\left(\left(\begin{array}{cc}1_{r} & 0 \\ 0 & 0\end{array}\right)\right)$, where $r=r_{p}(N)$ is the rank of $N$ over $\mathbb{F}_{p}$. It clearly follows that $R_{\alpha}^{n}(N)=0$ if $r>\alpha$, and $R_{\alpha}^{n}(N)$ is equal to the number of elements of $P_{n-\alpha, n-r}(p)$ if $r \leq \alpha$. It is well known that the last number can be computed by the formula

$$
\#\left(P_{n-\alpha, n-r}(p)\right)=\frac{\varphi_{n-r}(p)}{\varphi_{n-\alpha}(p) \varphi_{\alpha-r}(p)} \quad(r \leq \alpha)
$$

(see, e.g., [An87, Lemma 3.2.18]), where $\varphi_{a}(x)=(x-1)\left(x^{2}-1\right) \cdots\left(x^{a}-1\right)$ and $\varphi_{0}(x)=1$. Thus we arrive at the formula

$$
\begin{aligned}
& I\left(N, Q, T^{n}(p)\right)=\sum_{\alpha=r}^{n} \chi_{Q}\left(p^{\alpha}\right) p^{-\alpha k+\alpha(\alpha+1) / 2} \frac{\varphi_{n-r}(p)}{\varphi_{n-\alpha}(p) \varphi_{\alpha-r}(p)} \\
& =\chi\left(p^{r}\right) p^{-r k+r(r+1) / 2} \sum_{\beta=0}^{n-r} \frac{p^{\beta(\beta+1) / 2} \varphi_{n-r}(p)}{\varphi_{n-r-\beta}(p) \varphi_{\beta}(p)} \chi\left(p^{\beta}\right) p^{\beta(r-k)} \\
& =\chi_{Q}\left(p^{r}\right) p^{-r k+r(r+1) / 2} \prod_{\gamma=1}^{n-r}\left(1+\chi_{Q}(p) p^{\gamma+r-k}\right) \quad\left(r=r_{p}(N)\right),
\end{aligned}
$$

where we have exploited the identity

$$
\sum_{\beta=0}^{b} \frac{x^{\beta(\beta+1) / 2} \varphi_{b}(x)}{\varphi_{\beta}(x) \varphi_{b-\beta}(x)} z^{\beta}=\prod_{\gamma=1}^{b}\left(1+x^{\gamma} z\right)
$$

following by an easy induction similarly to the binomial formula. This proves formula (6.1).

Finally, formula (3.11) for $P=1$ and $T=T^{n}(p)$ with the notation (3.15) can be written in the form

$$
\begin{aligned}
& \left.\Theta^{n}(V, Z ; H, Q)\right|_{\mathbf{j}} T^{n}(p) \\
& =\sum_{r=0}^{n} \sum_{N \in C^{n}(Q / p), r_{p}(N)=r}\left(\chi_{Q}\left(p^{r}\right) p^{-r k+r(r+1) / 2} \prod_{\gamma=1}^{n-r}\left(1+\chi_{Q}(p) p^{\gamma+r-k}\right)\right) \\
& \times \mathbf{e}\left\{p V, Z, p^{-1} H, p^{-1} Q ; N\right\},
\end{aligned}
$$

which is, in fact, formula (6.2).

Proposition 6.2. Let $Q$ be the matrix of an integral nonsingular quadratic form in an even number of variables $m=2 k, q$ the level of $Q$, and $\chi_{Q}$ the corresponding Dirichlet character. Let $p$ be a prime number not dividing $q$. Then the set $A(Q, p)$ of automorphs is empty or not depending on 
whether $\chi_{Q}(p)=-1$ or $\chi_{Q}(p)=1$. In the latter case,

$$
\begin{aligned}
& \left.\Theta^{m}(V, Z ; H, Q)\right|_{\mathbf{j}} T^{m}(p) \\
& =p^{k} \prod_{i=1}^{k}\left(1+p^{-i}\right) \sum_{D \in A(Q, p) / \Lambda^{m}} \Theta^{m}\left(p D^{-1} V, Z ; p^{-1} H[D], p^{-1} Q[D]\right),
\end{aligned}
$$

where $\mathbf{j}=j_{Q}(M, Z)$ and $\Lambda^{m}=\mathrm{GL}_{m}(\mathbb{Z})$.

Proof. To examine the existence of automorphs of $Q$ with multiplier $p$, we use the language of quadratic spaces with quadratic form q modulo $p$ in the sense of [An87, Appendix 2]. Let $(\mathbf{V}, f)$ be a quadratic space over $\mathbb{F}_{p}=\mathbb{Z} / p \mathbb{Z}$ associated to the quadratic form $\mathbf{q}(X)$ modulo $p$. We recall that in this case $\mathbf{V}$ can be considered as the set of $m$-rows over $\mathbb{F}_{p}$ and $f: \mathbf{V} \rightarrow \mathbb{F}_{p}$ is a quadratic function given by

$$
f\left(\left(u_{1}, \ldots, u_{m}\right)\right)=\mathbf{q}\left(u_{1}, \ldots, u_{n}\right) \bmod p .
$$

A quadratic space $\left(\mathbf{V}^{\prime}, f^{\prime}\right)$ is called a subspace of $(\mathbf{V}, f)$ if $\mathbf{V}^{\prime} \subset \mathbf{V}$ and the restriction of $f$ to $\mathbf{V}^{\prime}$ is equal to $f^{\prime}$. The bilinear scalar product $\mathbf{u} \cdot \mathbf{v} \in \mathbb{F}_{p}$ of $\mathbf{u}, \mathbf{v} \in \mathbf{V}$ is defined by

$$
\mathbf{u} \cdot \mathbf{v}=f(\mathbf{u}+\mathbf{v})-f(\mathbf{u})-f(\mathbf{v}) .
$$

Two vectors $\mathbf{u}, \mathbf{v} \in \mathbf{V}$ with $\mathbf{u} \cdot \mathbf{v}=0$ are called orthogonal. A space $(\mathbf{V}, f)$ is said to be the direct (or orthogonal) sum of subspaces $\left(\mathbf{V}_{1}, f_{1}\right), \ldots,\left(\mathbf{V}_{t}, f_{t}\right)$,

$$
(\mathbf{V}, f)=\bigoplus_{i=1}^{t}\left(\mathbf{V}_{i}, f_{i}\right),
$$

if each $\mathbf{v}$ in $\mathbf{V}$ is uniquely of the shape $\mathbf{v}=\mathbf{v}_{1}+\cdots+\mathbf{v}_{t}$ and $f(\mathbf{v})=$ $f_{1}\left(\mathbf{v}_{1}\right)+\cdots+f_{t}\left(\mathbf{v}_{t}\right)$. A nonzero quadratic space with zero quadratic function is called isotropic. A quadratic space $(\mathbf{V}, f)$ is said to be degenerate if it contains an isotropic direct summand, otherwise it is called nondegenerate. If $p$ does not divide the determinant $\operatorname{det} \mathbf{q}=\operatorname{det} Q$ or the level of $Q$, then the corresponding quadratic space $(\mathbf{V}, f)$ is nondegenerate.

A set of vectors of the quadratic space $(\mathbf{V}, f)$ is said to be isotropic if the vectors are linearly independent and span an isotropic subspace. According to [An78, Lemma 5.2] or [An87, Proposition A.2.14], the number $i(\mathbf{V}, f ; r)$ of different isotropic sets of $r$ vectors in a nondegenerate quadratic space $(\mathbf{V}, f)$ over $\mathbb{F}_{p}$ can be computed by the formulas

$$
\begin{aligned}
& i(\mathbf{V}, f ; r) \\
& = \begin{cases}\left(p^{k}-\chi_{Q}(p)\right)\left(p^{k-1}+\chi_{Q}(p)\right) & \text { if } r=1, \\
p^{r(r-1) / 2}\left(p^{k}-\chi_{Q}(p)\right)\left(p^{k-r}+\chi_{Q}(p)\right) \prod_{i=1}^{r-1}\left(p^{2(k-i)}-1\right) & \text { if } r>1,\end{cases}
\end{aligned}
$$


where $k=m / 2$ and $\chi_{Q}(p)$ is the character $(2.15)-(2.17)$ of the form $\mathbf{q}$. In particular, for $r=m / 2=k$ we obtain

(6.12) $i(\mathbf{V}, f ; k)$

$$
= \begin{cases}0 & \text { if } \chi_{Q}(p)=-1, \\ (p-1)\left(p^{0}+1\right) & \text { if } \chi_{Q}(p)=1 \text { and } k=1, \\ p^{k(k-1) / 2}\left(p^{k}-1\right)\left(p^{0}+1\right) \prod_{i=1}^{k-1}\left(p^{2(k-i)}-1\right) & \text { if } \chi_{Q}(p)=1 \text { and } k>1 .\end{cases}
$$

Returning to automorphs, we first note that if they exist, the set $A(Q, p)$ is contained in a single double coset modulo $\Lambda=\Lambda^{m}$ :

$$
A(Q, p) \subset \Lambda D_{k}^{m} \Lambda \quad \text { with } \quad D_{k}^{m}=D_{k}^{m}(p)=\left(\begin{array}{cc}
1_{k} & 0 \\
0 & p \cdot 1_{k}
\end{array}\right) .
$$

In fact, if $D \in A(Q, p)$, then $\operatorname{det} D=p^{k}$, and so the elementary divisors of $D$ (see, e.g., $\left[\right.$ An87, §3.2.1]) have the form $p^{e_{1}}, \ldots, p^{e_{m}}$ where $0 \leq e_{1} \leq \cdots \leq$ $e_{m}$ and $e_{1}+\cdots+e_{m}=k$. Since $p$ does not divide $\operatorname{det} Q$, it follows from the properties of elementary divisors and the definition of automorphs that the elementary divisors of the matrix $p D^{-1}$, i.e., $p^{1-e_{m}}, \ldots, p^{1-e_{1}}$, coincide with those of $D$. Therefore, $0 \leq e_{\alpha} \leq 1$, and so $e_{1}=\cdots=e_{k}=0$ and $e_{k+1}=\cdots=e_{m}=1$, which proves (6.13). From (6.13) and (5.1) we can write

$$
A(Q, p)=\left\{D \in \Lambda D_{k}^{m} \Lambda \mid p^{-1} Q[D] \in \mathbb{E}^{m}\right\}=\Lambda D_{k}^{m} \Lambda \cap C^{m}(Q / p) .
$$

Thus, each automorph of $A(Q, p)$ can be written in the form $D=U D_{k}^{m} V$ with $U, V \in \Lambda$, and the rows $\mathbf{u}_{1}, \ldots, \mathbf{u}_{m}$ of ${ }^{t} U$ satisfy the condition

$$
\mathbb{E}^{m}={ }^{t} V^{-1} \mathbb{E}^{m} V \ni p^{-1 t} V^{-1} Q[D] V^{-1}=p^{-1 t}\left(U D_{k}^{m}\right) Q U D_{k}^{m},
$$

which means, as is not difficult to see, that the rows $\mathbf{u}_{1}, \ldots, \mathbf{u}_{k}$ considered modulo $p$ form an isotropic set of $k$ vectors of the space $\mathbf{V}=\left(\mathbb{F}_{p}\right)_{m}$ with quadratic function given by the form $\mathbf{q}$ modulo $p$. Hence $\chi_{Q}(p)=1$. Conversely, if $\chi_{Q}(p)=1$, then there is an isotropic set of $k$ integral $m$-rows considered modulo $p$, which can be completed to a matrix ${ }^{t} U \in \Lambda$ so that the matrix $U D_{k}^{m}$ belongs to $A(Q, p)$. This proves the first assertion.

Let us now assume that $\chi_{Q}(p)=1$ and consider the sum

$$
S(Q, p)=\sum_{D \in A(Q, p) / \Lambda} \Theta^{m}\left(p D^{-1} V, Z ; p^{-1} H[D], p^{-1} Q[D]\right)
$$

(with $\Lambda=\Lambda^{m}$ ). By (6.14) we have

$$
A(Q, p) / \Lambda=\left(\Lambda D_{k}^{m} \Lambda \cap C^{m}(Q / p)\right) / \Lambda .
$$

According to (6.4) with $n=m$ we can write

$$
\Lambda D_{k}^{m} \Lambda / \Lambda={ }^{t}\left\{\Lambda \backslash \Lambda D_{k}^{m} \Lambda\right\}=\left\{{ }^{t} \varepsilon(\mathbf{x}) D_{k}^{m} \mid \mathbf{x} \in P_{k, m}(p)\right\},
$$


where as above $\varepsilon(\mathbf{x})=\left(\frac{\mathbf{x}}{\mathbf{x}}\right) \in \mathrm{SL}_{m}(\mathbb{Z})$. The condition $D \in C^{m}(Q / p)$ for $D$ of the form ${ }^{t} \varepsilon(\mathbf{x}) D_{k}^{m}$ is clearly equivalent to ${ }^{t} \mathbf{x} \in C^{k}(Q / p)$. Hence

$$
A(Q, p) / \Lambda=\left\{{ }^{t} \varepsilon(\mathbf{x}) D_{k}^{m} \mid \mathbf{x} \in P_{k, m}(p),{ }^{t} \mathbf{x} \in C^{k}(Q / p)\right\} .
$$

Thus, the sum (6.15) can be written in the form

$$
\begin{gathered}
\begin{array}{c}
\text { 6.17) } S(Q, p)=\sum_{D \in\left(\Lambda D_{k}^{m} \Lambda / D \cap C^{m}(Q / p)\right) / \Lambda} \Theta^{m}\left(p D^{-1} V, Z ; p^{-1} H[D], p^{-1} Q[D]\right) \\
=\sum_{\mathbf{x},{ }^{t} \mathbf{x} \in{ }^{t} P_{k, m}(p) \cap C^{k}(Q / p)} \Theta^{m}\left(p\left(D_{k}^{m}\right)^{-1}{ }^{t} \varepsilon(\mathbf{x})^{-1} V, Z, p^{-1} H\left[{ }^{t} \varepsilon(\mathbf{x}) D_{k}^{m}\right] ;\right. \\
=\sum_{\mathbf{x},{ }^{t} \mathbf{x} \in{ }^{t} P_{k, m}(p) \cap C^{k}(Q / p)} \sum_{N \in \mathbb{Z}_{n}^{m}} \mathbf{e}\left\{p^{-1} Q\left[{ }^{t} \varepsilon(\mathbf{x}) D_{k}^{m}\left(N-p\left(D_{k}^{m}\right)^{-1}{ }^{t} \varepsilon(\mathbf{x})^{-1} V_{2}\right)\right] X\right. \\
+\sqrt{-1} p^{-1} H\left[{ }^{t} \varepsilon(\mathbf{x}) D_{k}^{m}\left(N-p\left(D_{k}^{m}\right)^{-1}{ }^{t} \varepsilon(\mathbf{x})^{-1} V_{2}\right)\right] Y \\
+2 \cdot{ }^{t} V_{1} \varepsilon(\mathbf{x})^{-1} p\left(D_{k}^{m}\right)^{-1} p^{-1} Q\left[{ }^{t} \varepsilon(\mathbf{x}) D_{k}^{m}\right] N \\
\left.-{ }^{t} V_{1} \varepsilon(\mathbf{x})^{-1} p\left(D_{k}^{m}\right)^{-1} p^{-1} Q\left[{ }^{t} \varepsilon(\mathbf{x}) D_{k}^{m}\right] p\left(D_{k}^{m}\right)^{-1}{ }^{t} \varepsilon(\mathbf{x})^{-1} V_{2}\right\} \\
\sum_{\mathbf{x},{ }^{t} \mathbf{x} \in{ }^{t} P_{k, m}(p) \cap C^{k}(Q / p)}{ }_{N \in \mathbb{Z}_{m}^{m}} \mathbf{e}\left\{p^{-1} Q\left[{ }^{t} \varepsilon(\mathbf{x}) D_{k}^{m} N-p V_{2}\right] X\right. \\
+\sqrt{-1} p^{-1} H\left[{ }^{t} \varepsilon(\mathbf{x}) D_{k}^{m} N-p V_{2}\right] Y+2{ }^{t}\left(p V_{1}\right) p^{-1} Q{ }^{t} \varepsilon(\mathbf{x}) D_{k}^{m} N \\
\left.-{ }^{t}\left(p V_{1}\right) p^{-1} Q\left(p V_{2}\right)\right\}
\end{array} \\
=\sum_{N \in \mathbb{Z}_{m}^{m}} \Phi(N) \mathbf{e}\left\{p V, Z, p^{-1} H, p^{-1} Q ; N\right\},
\end{gathered}
$$

where the notation (3.15) is again used, and where

$$
\Phi(N)=\sum_{\substack{\mathbf{x},{ }^{t} \mathbf{x} \in{ }^{t} P_{k, m}(p) \cap C^{k}(Q / p) \\ N \in{ }^{t} \varepsilon(\mathbf{x}) D_{k}^{m} \mathbb{Z}_{m}^{m}}} 1 .
$$

If $N={ }^{t} \varepsilon(\mathbf{x}) D_{m / 2}^{m} N^{\prime}$ with ${ }^{t} \mathbf{x} \in{ }^{t} P_{k, m}(p) \cap C^{k}(Q / p)$ and an integral matrix $N^{\prime}$, then the matrix

$$
\begin{aligned}
p^{-1} Q[N] & =p^{-1}{ }^{t} N^{\prime} D_{k}^{m} \varepsilon(\mathbf{x}) Q^{t} \varepsilon(\mathbf{x}) D_{k}^{m} N^{\prime} \\
& =p^{-1}{ }^{t} N^{\prime} D_{k}^{m}\left(\begin{array}{cc}
\mathbf{x} Q^{t} \mathbf{x} & \mathbf{x} Q^{t} \widehat{\mathbf{x}} \\
\widehat{\mathbf{x}} Q^{t} \mathbf{x} & \widehat{\mathbf{x}} Q^{t} \widehat{\mathbf{x}}
\end{array}\right) D_{k}^{m} N^{\prime}={ }^{t} N^{\prime}\left(\begin{array}{cc}
p^{-1} \mathbf{x} Q^{t} \mathbf{x} & \mathbf{x} Q^{t} \widehat{\mathbf{x}} \\
\widehat{\mathbf{x}} Q^{t} \mathbf{x} & p \widehat{\mathbf{x}} Q^{t} \widehat{\mathbf{x}}
\end{array}\right) N^{\prime}
\end{aligned}
$$

is clearly even. Hence, $\Phi(N)=0$ unless $N \in C^{m}(Q / p)$, and we can rewrite the expression (6.17) for the sum $S(Q, p)$ in the form

$$
S(Q, p)=\sum_{N \in C^{m}(Q / p)} \Phi(N) \mathbf{e}\left\{p V, Z, p^{-1} H, p^{-1} Q ; N\right\} .
$$


If $N \in \mathbb{Z}_{m}^{m}$ and the rank $r_{p}(N)$ of $N$ over $\mathbb{F}_{p}$ is $r$, then there is a matrix $U \in \Lambda=\mathrm{GL}_{m}(\mathbb{Z})$ such that $U N=D_{m-r}^{m} N^{\prime}$, where $D_{m-r}^{m}=D_{m-r}^{m}(p)$ and $N^{\prime}$ is an integral matrix. By transposition of the representative system (6.4) for $\alpha=m-r$, we conclude that $U^{-1} D_{m-r}^{m}={ }^{t} \varepsilon(\mathbf{z}) D_{m-r}^{m} U^{\prime}$, where $\mathbf{z} \in P_{r, m}(p)$ and $U^{\prime} \in \Lambda$. Therefore

$$
\begin{aligned}
& N=U^{-1} D_{m-r}^{m} N^{\prime}={ }^{t} \varepsilon(\mathbf{z}) D_{m-r}^{m} N^{\prime \prime} \\
& \quad\left(\mathbf{z} \in P_{r, m}(p), N^{\prime \prime}=U^{\prime} N^{\prime} \in \mathbb{Z}_{m}^{m}\right) .
\end{aligned}
$$

In addition, the condition $r_{p}(N)=r_{p}(\mathbf{x})$ is equivalent to the condition that the $r \times m$-block $N_{1}^{\prime \prime}$ of the matrix $N^{\prime \prime}=\left(\begin{array}{l}N_{1}^{\prime \prime} \\ N_{2}^{\prime \prime}\end{array}\right)$ satisfies

$$
r_{p}\left(N_{1}^{\prime \prime}\right)=r_{p}\left(\left(\begin{array}{c}
N_{1}^{\prime \prime} \\
p N_{2}^{\prime \prime}
\end{array}\right)\right)=r_{p}\left({ }^{t} \varepsilon(\mathbf{z})^{-1} N\right)=r .
$$

It is easy to see that the matrix $\mathbf{z}=z(N) \in P_{r, m}(p)$ in the factorization (6.20) is uniquely determined by $N$, and the condition $N \in C^{m}(Q / p)$ is equivalent to ${ }^{t} \mathbf{z} \in C^{r}(Q / p)$. We define a partial order on the union of the sets $P_{\alpha, m}(p)$ by setting $\mathbf{x} \preceq \mathbf{y}$ if each row of $\mathbf{x}$ is congruent modulo $p$ to a linear combination of rows of $\mathbf{y}$. This implies in particular that $r_{p}(\mathbf{x}) \leq r_{p}(\mathbf{y})$. If $\mathbf{z}=z(N) \preceq \mathbf{x} \in P_{\alpha, m}$, then there is an integral $r \times \alpha$-matrix $\mathbf{v}$ such that $\mathbf{v} \mathbf{x} \equiv \mathbf{z}(\bmod p)$. It is clear that $r_{p}(\mathbf{v})=r_{p}(\mathbf{z})=r$. One can easily see that there exists a matrix $V \in \Lambda$ satisfying $V \equiv\left(\begin{array}{cc}\mathbf{v} & 0 \\ * & *\end{array}\right)(\bmod p)$ and such that $V \varepsilon(\mathbf{x})=\varepsilon(\mathbf{z})$. Hence,

$$
\begin{aligned}
N & ={ }^{t} \varepsilon(\mathbf{z}) D_{m-r}^{m} N^{\prime \prime}={ }^{t} \varepsilon(\mathbf{x}){ }^{t} V D_{m-r}^{m} N^{\prime \prime} \\
& ={ }^{t} \varepsilon(\mathbf{x}) D_{m-\alpha}^{m}\left(D_{m-\alpha}^{m}\right)^{-1 t} V D_{m-r}^{m} N^{\prime \prime}={ }^{t} \varepsilon(\mathbf{x}) D_{m-\alpha}^{m} \tilde{N}
\end{aligned}
$$

with $\tilde{N} \in \mathbb{Z}_{m}^{m}$. Vice versa, if $N={ }^{t} \varepsilon(\mathbf{x}) D_{m-\alpha}^{m} \tilde{N}$, where $\mathbf{x} \in P_{\alpha, m}(p)$ and $\tilde{N} \in \mathbb{Z}_{m}^{m}$, then ${ }^{t} \mathbf{x} \tilde{N}_{1} \equiv{ }^{t} \mathbf{z} N_{1}(\bmod p)$, where $\tilde{N}_{1}$ is the upper $\alpha \times m$-block of $\tilde{N}$, which implies, by (6.20), that $\mathbf{z} \preceq \mathbf{x}$. These arguments show that the sum $(6.18)$ can be written in the form

$$
\Phi(N)=\sum_{\mathbf{x},{ }^{t} \mathbf{x} \in{ }^{t} P_{k, m}(p) \cap C^{k}(Q / p)} 1 .
$$

In order to compute this sum we return to the language of quadratic spaces. For $\mathbf{z} \in P_{r, m}(p)$ we denote by $V(\mathbf{z})$ the subspace of $\mathbf{V}$ spanned over $\mathbb{F}_{p}$ by the rows of $\mathbf{z}$. Then the condition $z(N) \preceq \mathbf{x}$ means that $V(z(N)) \subset V(\mathbf{x})$, and the condition ${ }^{t} \mathbf{x} \in C^{k}(Q / p)$ means that the space $V(\mathbf{x})$ is isotropic. It follows that $\Phi(N)$ is equal to the number of different isotropic subspaces of $\mathbf{V}$ of dimension $k$ which contain $V(z(N))$. Since $\chi_{Q}(p)=1$, there are $i(\mathbf{V}, f ; k) / \#\left(\mathrm{GL}_{k}\left(\mathbb{F}_{p}\right)\right)>0$ isotropic subspaces of dimension $k$, and every 
basis of $V(z(N))$ can be completed to a basis of an isotropic subspace of dimension $m / 2$ in exactly

$$
\frac{i(\mathbf{V}, f ; k)}{i(\mathbf{V}, f ; r)}=\left\{\begin{array}{lc}
1 & \text { if } r=k, \\
\left(p^{k(k-1) / 2-r(r-1) / 2}\left(p^{0}+1\right) \prod_{i=r}^{k-1}\left(p^{2(k-i)}-1\right)\right) /\left(p^{k-r}+1\right) & \text { if } 1 \leq r<k
\end{array}\right.
$$

ways. Two completed bases $\mathbf{v}_{1}, \ldots, \mathbf{v}_{r}, \mathbf{v}_{r+1}, \ldots, \mathbf{v}_{k}$ and $\mathbf{v}_{1}, \ldots, \mathbf{v}_{r}$, $\mathbf{v}_{r+1}^{\prime}, \ldots, \mathbf{v}_{k}^{\prime}$ with $k>r$ span the same space over $\mathbb{F}_{p}$ if and only if

$$
\left(\mathbf{v}_{1}, \ldots, \mathbf{v}_{r}, \mathbf{v}_{r+1}^{\prime}, \ldots, \mathbf{v}_{k}^{\prime}\right)=\left(\mathbf{v}_{1}, \ldots, \mathbf{v}_{r}, \mathbf{v}_{r+1}, \ldots, \mathbf{v}_{k}\right)\left(\begin{array}{cc}
1_{r} & B \\
0_{r}^{k-r} & D
\end{array}\right),
$$

where $B$ is an $r \times(k-r)$-matrix over $\mathbb{F}_{p}$ and $D \in \mathrm{GL}_{k-r}\left(\mathbb{F}_{p}\right)$. Therefore, an easy calculation shows that the number $\Phi(N)$ of different isotropic subspaces of $(\mathbf{V}, f)$ of dimension $k=m / 2$ which contain $V(z(N))$ is equal to

$$
\begin{aligned}
\frac{i(\mathbf{V}, f ; m / 2)}{i(\mathbf{V}, f ; r) p^{r(k-r)} \#\left(\mathrm{GL}_{k-r}\left(\mathbb{F}_{p}\right)\right)} & i(\mathbf{V}, f ; m / 2) \\
= & \frac{1(\mathbf{V}, f ; r) p^{r(k-r)} p^{(k-r)(k-r-1) / 2} \prod_{i=1}^{k-r}\left(p^{i}-1\right)}{i f} \\
& = \begin{cases}1 & \text { if } r=k, \\
\prod_{i=1}^{k-r}\left(p^{i-1}+1\right) & \text { if } r<k .\end{cases}
\end{aligned}
$$

Thus we finally get

$$
\Phi(N)= \begin{cases}1 & \text { if } \chi_{Q}(p)=1 \text { and } r(N)=k, \\ \prod_{i=1}^{k-r(N)}\left(p^{i-1}+1\right) & \text { if } \chi_{Q}(p)=1 \text { and } r(N)<k\end{cases}
$$

where $r(N)=r_{p}(N)$. Substituting this in (6.17), we obtain

$$
\begin{aligned}
& \quad \sum_{D \in A(Q, p) / \Lambda} \Theta^{m}\left(p D^{-1} V, Z ; p^{-1} H[D], p^{-1} Q[D]\right) \\
& =\sum_{N \in C^{m}(Q / p), r(N)=k} \mathbf{e}\left\{p V, Z, p^{-1} H, p^{-1} Q ; N\right\} \\
& \quad+\sum_{N \in C^{m}(Q / p), r(N)<k} \prod_{i=1}^{k-r(N)}\left(p^{i-1}+1\right) \mathbf{e}\left\{p V, Z, p^{-1} H, p^{-1} Q ; N\right\} .
\end{aligned}
$$


On the other hand, by formula (6.2) with $n=m$, we have

$$
\begin{aligned}
=\sum_{N \in C^{m}(Q / p)}\left(\chi_{Q}\left(p^{r(N)}\right) p^{-r(N) k+r(N)(r(N)+1) / 2}\right. & \\
& \left.\times \prod_{\gamma=1}^{m-r(N)}\left(1+\chi_{Q}(p) p^{\gamma+r(N)-k}\right)\right) \\
& \times \mathbf{e}\left\{p V, Z, p^{-1} H, p^{-1} Q ; N\right\} .
\end{aligned}
$$

Let us now compare this formula with (6.24). If $r(N)=r_{p}(N)=m / 2=k$, then the coefficient of $\mathbf{e}\left\{p V, Z, p^{-1} H, p^{-1} Q ; N\right\}$ on the right side of (6.25) is

$$
\begin{aligned}
p^{-r(N) k+r(N)(r(N)+1) / 2} \prod_{\gamma=1}^{m-r(N)}\left(1+p^{\gamma+r(N)-k}\right) & \\
& =p^{-k(k-1) / 2} \prod_{\gamma=1}^{k}\left(1+p^{\gamma}\right)=p^{k} \prod_{i=1}^{k}\left(1+p^{-i}\right),
\end{aligned}
$$

but if $r(N)<m / 2=k$, on replacing of $\gamma$ by $i=\gamma+r(N)-k$ the coefficient can be written in the form

$$
\begin{aligned}
& p^{-r(N) k+r(N)(r(N)+1) / 2} \prod_{i=1+r(N)-k}^{k}\left(1+p^{i}\right) \\
& =p^{-r(N) k+r(N)(r(N)+1) / 2}\left(\prod_{i=1}^{k}\left(1+p^{i}\right)\right)\left(\prod_{i=1+r(N)-k}^{0}\left(1+p^{i}\right)\right) \\
& =p^{-r(N) k+r(N)(r(N)+1) / 2+k(k+1) / 2}\left(\prod_{i=1}^{k}\left(1+p^{-i}\right)\right)\left(\prod_{j=1}^{k-r(N)}\left(1+p^{1-j}\right)\right),
\end{aligned}
$$

where in the last product we have set $i=1-j$. Similarly to the first product, we can rewrite the second product in the form

$$
\begin{aligned}
\prod_{j=1}^{k-r(N)}\left(1+p^{1-j}\right) & =\prod_{j=1}^{k-r(N)} p^{1-j}\left(p^{j-1}+1\right) \\
& =p^{-(k-r(N))(k-r(N)-1) / 2} \prod_{j=1}^{k-r(N)}\left(p^{j-1}+1\right) .
\end{aligned}
$$

Thus, the whole coefficient is equal to 


$$
\begin{aligned}
& p^{-r(N) k+r(N)(r(N)+1) / 2+k(k+1) / 2}-(k-r(N))(k-r(N)-1) / 2 \\
& \times\left(\prod_{i=1}^{k}\left(1+p^{-i}\right)\right)\left(\prod_{j=1}^{k-r(N)}\left(1+p^{1-j}\right)\right) \\
&= p^{k}\left(\prod_{i=1}^{k}\left(1+p^{-i}\right)\right)\left(\prod_{j=1}^{k-r(N)}\left(1+p^{1-j}\right)\right) .
\end{aligned}
$$

Comparing the corresponding coefficients, we arrive at (6.10).

It is not difficult to deduce from (5.6) with $n=m$ and $N=0_{m}^{m}$ and (4.9) that for $\chi_{Q}(p)=1$ the set $A(Q, p) / \Lambda^{m}$ of classes of automorphs of $Q$ with multiplier $p$ modulo the group $\Lambda^{m}=\mathrm{GL}_{m}(\mathbb{Z})$ consists of

$$
\#\left(A(Q, p) / \Lambda^{m}\right)=\prod_{i=0}^{k-1}\left(1+p^{i}\right)
$$

elements.

In order to consider the action of the Hecke operators corresponding to the elements $T^{n}(p)$ with $n \neq m$, we have to compute the images and inverse images of these elements under Zharkovskaya homomorphisms.

Lemma 6.3. Let $n>r \geq 0$ and let $p$ be a prime not dividing $\operatorname{det} Q$. Then

$$
\Psi_{Q}^{n, r}\left(T^{n}(p)\right)=\prod_{i=1}^{n-r}\left(1+\chi_{Q}(p) p^{i+r-k}\right) T^{r}(p),
$$

where $k=m / 2$; in particular, if $r<k \leq n$ and $\chi_{Q}(p)=-1$, then $\Psi_{Q}^{n, r}\left(T^{n}(p)\right)=0$, and $T^{r}(p) \notin \Psi_{Q}^{n, r}\left(\mathcal{H}_{0}^{n}(q)\right)$; otherwise, the product in $(6.26)$ is not zero and $T^{r}(p) \in \Psi_{Q}^{n, r}\left(\mathcal{H}_{0}^{n}(q)\right)$.

Proof. Let us present the element $T^{n}(p)$ in the shape (3.2) with triangular representatives $M_{\alpha}$ of the form (4.7) for $r=n-1 \geq 1$. Then it is not hard to see that $D_{\alpha}^{\prime \prime}= \pm 1$ or $\pm p$, and that in the notation of the definition of the Zharkovskaya homomorphism for $r=n-1$ we have the expansions

$$
\begin{aligned}
\sum_{\alpha, D_{\alpha}^{\prime \prime}= \pm 1}\left(\Gamma_{0}^{n-1}(q) M_{\alpha}^{\prime}\right) & =T^{n-1}(p), \\
\sum_{\alpha, D_{\alpha}^{\prime \prime}= \pm p}\left(\Gamma_{0}^{n-1}(q) M_{\alpha}^{\prime}\right) & =p^{n} T^{n-1}(p) .
\end{aligned}
$$

Hence

$$
\begin{aligned}
\Psi_{Q}^{n, n-1}\left(T^{n}(p)\right) & =\left(1+p^{n}\left(\chi_{Q}(p) p^{k}\right)^{-1}\right) T^{n-1}(p) \\
& =\left(1+\chi_{Q}(p) p^{n-k}\right) T^{n-1}(p)
\end{aligned}
$$


This formula clearly remains true also for $n=1$ with $T^{0}(p)=1$. Then (6.26) follows by iteration. If $r<k \leq n$ and $\chi_{Q}(p)=-1$, then the factor on the right corresponding to $i=k-r$ (and only in this case) equals zero. The rest is clear: since the sets of generators (3.3) are algebraically independent, it follows that each homogeneous element of $\mathcal{H}_{0}^{n}(q)$ with multiplier $p$ is a constant multiple of $T^{n}(p)$.

We can finally prove the following formulas for the images of thetafunctions under the action of Hecke operators corresponding to elements $T^{n}(p)$.

TheOREM 6.3. Let $Q$ be a nonsingular even matrix of an even order $m=2 k$ with signature $(s, l), q$ the level of $Q$, and $\chi_{Q}$ the character of the corresponding quadratic form; let $H \in \mathbb{M}(Q)$, and $P$ be a polynomial harmonic form on $\mathbb{C}_{n}^{m}$ of weight $\left(d, d^{\prime}\right)$ relative to the pair $Q, H$. Finally, let $p$ be a rational prime not dividing $q$. Then the following explicit formulas hold for the action of the Hecke operator $\left.\right|_{\mathbf{j}} T^{n}(p)$ with automorphic factor $\mathbf{j}=j_{Q, P}$ of the form (2.14) on the theta-function (2.8) of genus $n$ with harmonic coefficient form $P$ :

$$
\begin{aligned}
\left.\Theta_{P}^{n}(V, Z ; H, Q)\right|_{\mathbf{j}} T^{n}(p)=p^{n(n+1) / 2-k n} \xi(n, m) & \\
\times & \sum_{D \in A(Q, p) / \Lambda^{m}} \Theta_{P \mid p^{-1} D}^{n}\left(p D^{-1} V, Z ; p^{-1} H[D], p^{-1} Q[D]\right)
\end{aligned}
$$

if $\chi_{Q}(p)=1$, where

$$
\begin{aligned}
\xi(n, m) & = \begin{cases}\prod_{i=1}^{n-k}\left(1+p^{-i}\right) & \text { if } n>k, \\
1 & \text { if } n=k, \\
\prod_{i=1}^{k-n}\left(1+p^{i-1}\right) & \text { if } n<k,\end{cases} \\
\left(P \mid p^{-1} D\right)(X) & =P\left(p^{-1} D X\right),
\end{aligned}
$$

and

$$
\left.\Theta_{P}^{n}(V, Z ; H, Q)\right|_{\mathbf{j}} T^{n}(p)=0 \quad \text { if } \chi_{Q}(p)=-1 \text { and } n \geq k \text {. }
$$

Proof. If $\chi_{Q}(p)=1$, then by Proposition 6.2 the set $A(Q, p)$ is not empty and the relation (6.10) holds. In this case, by Lemma 6.3, the elements $T=T^{n}(p)$ satisfy the condition (5.5) of Proposition 5.2, and according to that proposition and Proposition 6.2, for every $n=1,2, \ldots$,

$$
\begin{aligned}
\left.\Theta_{P}^{n}(V, Z ; H, Q)\right|_{\mathbf{j}} T^{n}(p)= & \sum_{D \in A(Q, p) / \Lambda^{m}} I\left(D, Q, \Psi_{Q}^{n, m}\left(T^{n}(p)\right)\right) \\
& \times \Theta_{P \mid p^{-1} D}^{n}\left(p D^{-1} V, Z ; p^{-1} H[D], p^{-1} Q[D]\right) .
\end{aligned}
$$


Then (6.27) follows from (6.10), (6.26) and (6.29) by considering the four cases: $n>m, m>n>k, n=k$, or $n<k$. For example, if $n>m$, then

$$
\begin{aligned}
I\left(D, Q, \Psi_{Q}^{n, m}\left(T^{n}(p)\right)\right) & =I\left(D, Q,\left(\prod_{i=1}^{n-m}\left(1+p^{i+k}\right)\right) T^{m}(p)\right) \\
& =\left(p^{k} \prod_{i=1}^{k}\left(1+p^{-i}\right)\right)\left(\prod_{i=1}^{n-m}\left(1+p^{i+k}\right)\right) \\
& =p^{k+(n-m)(n-m+1) / 2+(n-m) k} \prod_{i=1}^{n-m}\left(1+p^{-i}\right),
\end{aligned}
$$

which proves (6.27) in this case. The other cases can be handled similarly.

If $\chi_{Q}(p)=-1$, then by Lemma 6.3 the condition (5.5) of Proposition 5.2 for $T=T^{n}(p)$ is satisfied unless $n<k=m / 2$. In this case, looking at (6.2), one can note that the rank $r(N)=r_{p}(N)$ modulo $p$ of each matrix $N \in C^{n}(Q, p)$ is less than $k=m / 2$, since otherwise among the rows of the matrix ${ }^{t} N$ modulo $p$ there are $k$ rows which form an isotropic set of $k$ vectors in $(\mathbf{V}, f)$, contrary to $(6.12)$. Therefore, each term of the sum on the right of (6.1) and (6.2) contains a product including a factor with $n-r(N) \geq \gamma=$ $k-r(N) \geq 1$, which is zero. In particular, $I\left(N, Q, T^{m}(p)\right)=0$. Then (6.28) follows from Proposition 5.2.

Appearance of a power of $p$ on the right of (6.27) in contrast to similar formulas for theta-series of positive definite quadratic forms obtained in [An80] and [An87] is due to different normalization of Hecke operators.

\section{References}

[An79] A. N. Andrianov, Multiplicative arithmetic of Siegel modular forms, Uspekhi Mat. Nauk 34 (1979), no. 1, 67-135 (in Russian); English transl.: Russian Math. Surveys 34 (1979), 75-148.

[An80] - Action of Hecke operator T(p) on theta series, Math. Ann. 247 (1980), 245254.

[An86] - , The action of Hecke operators on nonhomogeneous theta series, Mat. Sb. (N.S.) 131 (1986), 275-292 (in Russian); English transl.: Math. USSR-Sb. 59 (1988), 269-285.

[An87] -, Quadratic Forms and Hecke Operators, Grundlehren Math. Wiss. 286, Springer, Berlin, 1987.

[An95] - Symmetries of harmonic theta-functions of integral quadratic forms, Uspekhi Mat. Nauk 50 (1995), no. 4, 3-44 (in Russian); English transl.: Russian Math. Surveys 50 (1991), 661-700.

[An96] -, Harmonic theta-functions and Hecke operators, Algebra i Analiz 8 (1996), no. 5, 1-31 (in Russian); English transl.: St. Petersburg Math. J. 8 (1997), 695720. 
[An06] A. N. Andrianov, Zeta-functions of orthogonal groups of integral positive definite quadratic forms, Uspekhi Mat. Nauk 61 (2006), no. 6, 3-44 (in Russian); English transl.: Russian Math. Surveys 61 (2006), 661-700.

[Fr81] E. Freitag, Eine Bemerkung zu Andrianovs expliziten Formeln für die Wirkung der Heckeoperatoren auf Thetareihen, in: E. B. Christoffel (Aachen/Monschau, 1979), Birkhäuser, 1981, 336-351.

[Si44] C. L. Siegel, On the theory of indefinite quadratic forms, Ann. of Math. 45 (1966), $577-622$.

St. Petersburg Division of the Steklov Mathematical Institute of the Russian Academy of Sciences (POMI)

Fontanka 27

191023 St. Petersburg, Russia

E-mail: anandr@pdmi.ras.ru

anatoli.andrianov@gmail.com 